\title{
Analysis of Supply Contracts with Commitments and Flexibility
}

\author{
Yehuda Bassok, ${ }^{1}$ Ravi Anupindi ${ }^{2}$ \\ ${ }^{1}$ Marshall School of Business, University of Southern California, Los Angeles, California 90089 \\ ${ }^{2}$ Ross School of Business, University of Michigan, Ann Arbor, Michigan 48109
}

Received 10 February 2006; revised 28 March 2008; accepted 29 March 2008

DOI 10.1002/nav.20300

Published online 4 June 2008 in Wiley InterScience (www.interscience.wiley.com).

\begin{abstract}
In this article we address an important class of supply contracts called the Rolling Horizon Flexibility (RHF) contracts. Under such a contract, at the beginning of the horizon a buyer has to commit requirements for components for each period into the future. Usually, a supplier provides limited flexibility to the buyer to adjust the current order and future commitments in a rolling horizon manner. We present a general model for a buyer's procurement decision under RHF contracts. We propose two heuristics and derive a lower bound. Numerically, we demonstrate the effectiveness of the heuristics for both stationary and non-stationary demands. We show that the heuristics are easy to compute, and hence, amenable to practical implementation. We also propose two measures for the order process that allow us to (a) evaluate the effectiveness of RHF contracts in restricting the variability in the orders, and (b) measure the accuracy of advance information vis-a-vis the actual orders. Numerically we demonstrate that the order process variability decreases significantly as flexibility decreases without a dramatic increase in expected costs. Our numerical studies provide several other managerial insights for the buyer; for example, we provide insights into how much flexibility is sufficient, the value of additional flexibility, the effect of flexibility on customer satisfaction (as measured by fill rate), etc. ㅇ 2008 Wiley Periodicals, Inc. Naval Research Logistics 55: 459-477, 2008
\end{abstract}

Keywords: supply contracts; flexibility; inventory

\section{INTRODUCTION}

A major part of every manufacturing and distribution activity is to ensure a smooth supply of raw materials and components needed to produce and assemble the final product in a cost-effective manner. This is especially important because, increasingly, in many industries the cost of raw materials/components constitutes approximately $60-70 \%$ of the cost of an end-product. A manufacturer needs to make sourcing, supplier selection, and business allocation decisions in the presence of several types of uncertainties in supply and demand. Supply contracts can play an important role in managing these uncertainties.

Different supply contracts will have different impact on the behavior of the parties involved, risks imposed on them, and the resulting efficiencies; see for example, discussions in Anupindi and Bassok [4]. Fundamentally, a buyer faced with stochastic demand takes on the demand risk by keeping some safety stock; this is unavoidable as long as the buyer has to place orders before observing the demand. A supplier's risk is due to the uncertainty in the order process which may affect its

Correspondence to: R. Anupindi (anupindi@umich.edu) production costs. Suppliers wary of taking the risk might ask for firm commitments from the buyer, which in turn imposes risk on the buyer. In the classical uncapacitated newsvendor problem with no commitments the order process has the same level of uncertainty as the demand process; such "newsvendor contracts" may not be acceptable to the supplier. Realistic contracts should strike a balance between the risks faced by a supplier (of uncertainty in orders) and risks faced by the buyer (of making commitments to purchase certain quantities).

Various types of contracts can be proposed that try to achieve this balance between risk sharing and commitment. In this article, we analyze one such class of contracts popularly used in the industry, called a rolling horizon flexibility (RHF) contract. Under such a contract, at the beginning of the horizon a buyer has to forecast and make commitments for components for each period into the future. Usually, a supplier provides limited flexibility to the buyer to adjust the current order and future commitments in a rolling horizon manner. Consider the following example: a supplier allows a 5\% flexibility to adjust the current period order, $10 \%$ flexibility to adjust the commitment one-period from now, and $20 \%$ flexibility to adjust the commitment two periods from now. We illustrate the dynamics of such a RHF model in 


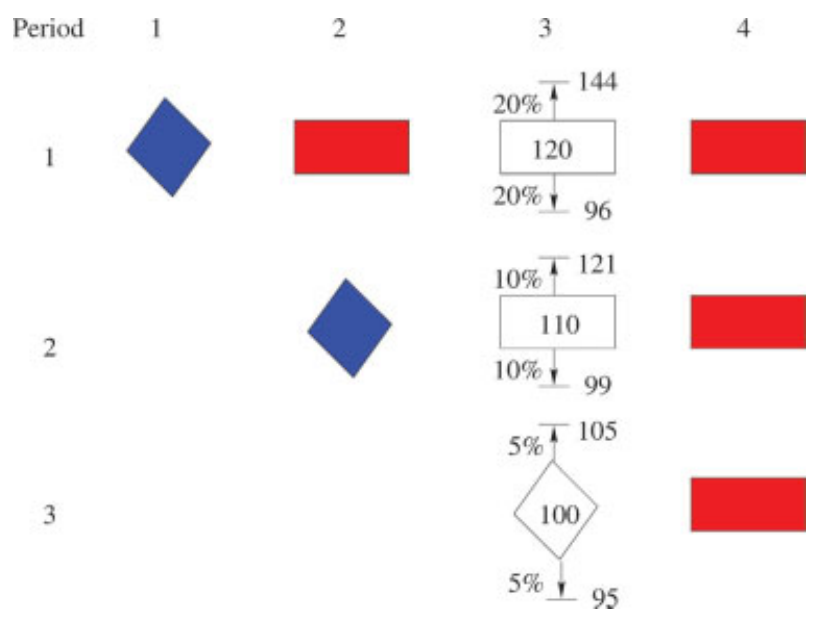

Figure 1. The Dynamics of a RHF Contract. [Color figure can be viewed in the online issue, which is available at www.interscience.wiley.com.]

Fig. 1 where the current period is represented by a diamond and future periods by a rectangle. Suppose that the commitment for period 3 in period 0 was 120 . In period 1 , this commitment can be adjusted by $20 \%$. Thus the period 3 commitment made in period 1 could be between $[96,144]$. Say, the commitment updated in period 1 for period 3 is 110. In period 2, the commitment made for period 3 can be adjusted by $10 \%$; that is, the new commitment could be between [99, 121]. Say the buyer chooses 100 . In period 3, the buyer is allowed to actually purchase within $5 \%$ of previous commitment of 100, i.e., between 95 and 105 .

Usually these types of contracts provide the manufacturer with a high level of long-term and a low level of short-term flexibility. The short-term flexibility is low because the supplier may have limited means to adjust quantities within the production lead-time. Bassok et al. [8] discuss an example of such a contract used by the printer division of a major computer manufacturer. Sun Microsystems uses similar contracts for the purchase of various components [12]. Tsay and Lovejoy [21] present several examples of companies in electronic and nonelectronic industries that use RHF contracts.

RHF contracts are useful under two situations. First, suppose a supplier's productions costs are affected by the variability in the order process. Note that the buyer's operating costs are clearly affected by the variability of the demand process. The type of flexibility contract observed will then depend on the cost of variability to each player and the channel structure (for e.g., centralized vs. decentralized). For example, Anupindi [1] shows that a flexibility contract will be observed under a cooperative game theoretic setting as long as both players have costs of variability. Furthermore, the variability in the order process could be adversely affected by a forecasting process adopted by a buyer under either stationary [11] or nonstationary [15] demands. Thus, such contracts could be of potentially greater value to a supplier when a buyer adopts some forecasting process for market demand. Second, suppose that there is asymmetric information between a buyer and a seller regarding market demand. In this case a buyer may have an incentive to mis-state his intended purchase. Flexibility contracts (by limiting flexibility unlike in a newsvendor contract) make a buyer partially responsible for any advance information regarding orders that he passes to the supplier.

In this article we present a general model for an RHF contract for procurement. Our article focuses on the analysis of the buyer's procurement problem. Informally, in an RHF contract a buyer needs to compute the initial commitments for the entire horizon knowing that he has the flexibility to (a) adjust the actual purchase in a period and (b) update future commitments, such that the expected costs are minimized. An optimal solution to the general RHF contract is unknown and perhaps complex. We present two heuristics. One of them is based on the concept of open loop feedback control (OLFC). The second is based on a specialization of the RHF contract, called a zero leadtime flexibility (ZLF) contract. Under a ZLF contract, a supplier offers some flexibility to adjust the actual order quantity in any given period based on a priori commitment for that period but does not allow the future commitments to be adjusted; because the adjustment to obtain the order quantity for a period is done in that period, we call this a ZLF contract. We discuss the optimal policy structure for a ZLF contract. We then show that optimal solutions to appropriately derived ZLF contracts give us an upper and a lower bound on the optimal costs under the RHF contract. Furthermore, we propose two metrics for the order process that allow us to measure the variability in the order process and the nature of advance information provided through the commitments.

Our objective, in addition to studying the policy structures and algorithms for the various contracts, is two-fold: (a) to provide technical insights into the problem and solution structures that enable fast computation, and (b) to provide managerial insights, whenever possible, regarding the performance of such contracts. From a managerial perspective, we are primarily interested in answers to the following types of questions: (i) How much flexibility of any given type is sufficient? (ii) What is the value of an additional (say, 5\%) flexibility? (iii) What is the impact of various types of contracts on the variability of the order process? (iv) What is the nature of advance information provided by an RHF contract? In addition, we discuss the appropriateness of an RHF contract for various demand patterns. For example, we show that for several cases (depending on the demand patterns and cost structure) for every RHF contract there is a ZLF contract that results in the same expected costs to the buyer but a significantly lower order process variability. When this happens, a 
buyer should be indifferent between RHF and ZLF contracts while the supplier should prefer the latter. Clearly, answers to such questions may depend on the cost and demand parameters. Therefore, we believe that the technical insights are also important as they allow us to quickly evaluate complex contracts and test various what-if scenarios.

The rest of the article is organized as follows. In Section 2, we present a brief review of literature on supply contracts with quantity commitments. In Section 3, we present a general model for the RHF contract. We also present two measures to compare the order process characteristics for the various contracts. In Section 4, we develop two heuristics for the general RHF contract as well a lower bound. First we derive the OLFC optimal solution to the RHF contract and give a constructive algorithm. Then we construct the ZLF contract and show that the optimal solution to an appropriately defined ZLF contract gives us an upper bound to the solution of the RHF contract. Finally, we derive a lower bound (based on a variant of the ZLF contract) to the optimal solution for the RHF contract. We discuss the optimal policy structure and algorithms for the ZLF contract in Section 5. We present computational studies and managerial insights in Section 6. We conclude in Section 7. All algorithms appear in Appendix A; Appendix B contains proofs of all results; Appendix $\mathrm{C}$ outlines the implementation of various heuristics and the lower bound; and, Appendix D presents a new heuristic based on the two proposed in the main article.

\section{LITERATURE REVIEW}

Quantity commitment contracts were first analyzed by Anupindi [1]. He discussed a contract similar to the ZLF contract described here but that differed in the way flexibility is modeled. His model allowed for non-negative adjustments of committed quantities and also supplier's responsiveness to deliver on the adjustments. That is, previously committed quantities are delivered in the respective periods whereas dynamic adjustments may or may not be delivered in the same period. In contrast we allow for positive and negative adjustments but do not model supplier responsiveness explicitly. For the case of stationary commitments, Anupindi and Akella [2] show that the commitment contract reduces the variance (as compared to the newsvendor model) of the order process. Moinzadeh and Nahmias [16] study a continuous review equivalent of a similar contract with stationary commitments under a diffusion approximation of demand. Scheller-Wolf and Tayur [18] show how quantity contracts can be used to reduce international risk under exchange rate uncertainty. Supply contracts with quantity commitments and flexibility to adjust, modeled through purchase of real options, is analyzed by Barnes-Schuster et al. [5]. Although the contracts mentioned thus far allow for periodical commitments, supply contracts with total volume commitment for single products and total dollar volume commitments for multiple products, respectively, are analyzed by Bassok and Anupindi [6], Anupindi and Bassok [3], and Chen and Krass [10].

Bassok et al. [8] present a heuristic to determine the nearoptimal purchasing quantity and the near-optimal periodical forecasts for an RHF contract. They assume a RHF contract with stationary flexibilities and their heuristic assumes that demands are normally distributed. They show that the heuristic performs quite well under realistic assumptions. Tsay and Lovejoy [21] — henceforth referred to as TL99 — analyze RHF-type contracts for a multi-echelon environment. In their model each stage in the supply chain is subject to an RHF contract. The downstream stage faces stochastic market demand which is a nonstationary process modeled as an exponentially weighted moving average process. They present heuristics for operation of various stages of the supply chain. In extensive numerical studies they evaluate the impact of demand variance, exponential smoothing factor, and flexibility parameters on inventory, costs, fill-rate, and variability in order and forecast processes. Our work is similar in many respects but focuses on a more in-depth analysis of a single stage system that faces nonstationary market demand. Both pieces of work are based on heuristics. For the last stage, TL99 present several heuristics (all modifications based on open loop feedback control mechanism) and select one for further analysis based on comparison between the heuristics. In contrast, we present two heuristics (the first is similar to the chosen heuristic in TL99) and also a lower bound. We compare the heuristics to one another and to the lower bound. We show that the relative performance of the heuristics depend on the problem parameters. We see that the end-of-horizon salvage value critically impacts the performance of various heuristics. Furthermore, we define two performance metrics for the order process. The order process variability measure is similar to the one described in TL99 except that while they measure standard deviation we report the coefficient of variation. In addition, we define a metric to measure the reliability of the advance information provided to the supplier through the commitment process. While our numerical studies confirm several findings in TL99, in addition, we give insights into the nature/reliability of advance information, performance of various heuristics, gap with respect to a lower bound, and appropriateness of contracts for various scenarios.

Tsay [20] demonstrates the role of flexibility contracts to achieve channel coordination in a single period model with asymmetric information between one buyer and one supplier. Lee et al. [15] mention the use of a RHF-type contract as an instrument to share forecast information, risk, and flexibility between a buyer and a supplier in the computer and retail companies. They suggest these contracts as a way to counter 
the gaming strategies that lead to information distortion in the supply chain.

Anupindi and Bassok [4] provide a general framework for the study of supply contracts and present a brief overview of supply contracts with quantity commitments. A recent survey of research in supply contracts appears in Tsay et al. [22].

More recent research has focused on computational approaches to solving the complex optimization arising from the use of RHF contracts. For example, Tal et al. [19] present a robust optimization approach to solving RHF contracts with a min-max objective.

\section{A GENERAL MODEL}

A finite horizon RHF contract is described as follows. At the beginning of the horizon, the buyer gives an initial commitment $Q_{1, i}$ for all periods $i=1, \ldots, T$ in the horizon. The supplier offers flexibility to adjust the commitments for future periods and actual orders, upwards or downwards, in any period. Let $\underline{\alpha}_{i, j}, i \leq j$, be the fraction by which the buyer may adjust period $j$ commitment (or actual order if $j=i$ ) downward at the beginning of period $i$. Similarly, let $\bar{\alpha}_{i, j}$, $i \leq j$, be the fraction by which the buyer may adjust period $j$ commitment (or actual order if $j=i$ ) upward at the beginning of period $i$. Clearly, $\underline{\alpha}_{i, j}=0$ and $\bar{\alpha}_{i, j}=0$ for $i>j$. Let $Q_{t, t+i}$ denote the commitment for period $t+i$ made in period $t$. Then,

- the actual order quantity in period $t, Q_{t, t}$ given the updated commitments and available flexibility is constrained to be as follows:

$$
\left(1-\underline{\alpha}_{t, t}\right) Q_{t-1, t} \leq Q_{t, t} \leq\left(1+\bar{\alpha}_{t, t}\right) Q_{t-1, t}
$$

- the commitment $Q_{t, t+i}$ for a future period, $t+i$, given the previous commitment for this period $Q_{t-1, t+i}$ is constrained by the corresponding downward and upward flexibilities to be as follows:

$$
\left(1-\underline{\alpha}_{t, t+i}\right) Q_{t-1, t+i} \leq Q_{t, t+i} \leq\left(1+\bar{\alpha}_{t, t+i}\right) Q_{t-1, t+i} .
$$

The upward and downward flexibilities may be specified as two matrices $[\underline{\alpha}]$ and $[\bar{\alpha}]$ with entries $\underline{\alpha}_{i, j}$ and $\bar{\alpha}_{i, j}$, respectively. This characterization of an RHF contract is most general because it allows for asymmetric and non-stationary flexibility for adjustments. Usually, most RHF contracts have the property that $\underline{\alpha}_{t_{1}, t_{1}+j}=\underline{\alpha}_{t_{2}, t_{2}+j}$, and $\bar{\alpha}_{t_{1}, t_{1}+j}=\bar{\alpha}_{t_{2}, t_{2}+j}$ for all $t_{1}, t_{2}$, and $j$. That is, the flexibility offered for a future period depends only in the number periods into the future.

The buyer faces a stochastic demand in each period; let $D_{t}$ represent the random variable for demand with a probability density function of $f_{t}(\cdot)$ and a cumulative density function of
$F_{t}(\cdot)$. Observe that we allow for demands to be nonstationary. We only assume that they be independent across periods. ${ }^{1} \mathrm{At}$ the beginning of each period $t$, he observes his inventory position $x_{t}$ and orders a quantity $Q_{t, t}$ for period $t$ (constrained as in (1)). In addition, he updates the commitment for the future periods (constrained as in (2)). The quantity ordered for the current period is delivered immediately. Subsequently, the buyer sees the period $t$ demand and satisfies it from stock on-hand as much as possible. Any excess demand is backordered. The marginal holding, penalty and purchase costs per period are $h, p$, and $c$, respectively. In the last period, any excess inventory may be salvaged at a unit price of $s \geq 0$.

In every period $t$ the buyer needs to determine the actual order quantity to purchase and update commitments for future periods so as to minimize total expected costs. This problem can be formulated as a stochastic dynamic program. Let $x_{t}$ be the starting inventory in period $t$ before ordering. The single period expected holding and penalty costs when the total quantity on hand at the beginning of period $t$ after receiving the orders is $x_{t}+Q_{t, t}$ is written as:

$$
\begin{aligned}
L_{t}\left(x_{t}+Q_{t, t}\right)= & h \mathbf{E}\left[\max \left\{x_{t}+Q_{t, t}-D_{t}, 0\right\}\right] \\
& +p \mathbf{E}\left[\max \left\{D_{t}-x_{t}-Q_{t, t}, 0\right\}\right] .
\end{aligned}
$$

Then the buyer's problem in period $t=2, \ldots, T$ is:

$$
\begin{aligned}
& \text { (RHF) } T C_{t}^{*}\left(x_{t}, Q_{t-1, t}, \ldots, Q_{t-1, T}\right)= \\
& \min _{Q_{t, t}, \ldots, Q_{t, T}} T C_{t}\left(Q_{t, t}, \ldots, Q_{t, T} \mid x_{t}, Q_{t-1, t}, \ldots, Q_{t-1, T}\right)
\end{aligned}
$$

subject to

$$
\left(1-\underline{\alpha}_{t, i}\right) Q_{t-1, i} \leq Q_{t, i} \leq\left(1+\bar{\alpha}_{t, i}\right) Q_{t-1, i} \quad i=t, \ldots, T
$$

where,

$$
\begin{aligned}
& T C_{t}\left(Q_{t, t}, \ldots, Q_{t, T} \mid x_{t}, Q_{t-1, t}, \ldots, Q_{t-1, T}\right)= \\
& \quad c Q_{t, t}+L_{t}\left(x_{t}+Q_{t, t}\right)+\mathbf{E}_{D_{t}}\left\{T C_{t+1}^{*}\left(x_{t+1}, Q_{t, t}, \ldots, Q_{t, T}\right)\right\}
\end{aligned}
$$

is the expected cost from period $t$ through $T$ given a starting inventory of $x_{t}$ and commitments $Q_{t-1, t}, \ldots, Q_{t-1, T}$ made in period $t-1$, and $x_{t+1}=x_{t}+Q_{t, t}-D_{t}$ for $t=1, \ldots, T$. Define the terminal cost $T C_{T+1}^{*}\left(x_{T+1}\right)=-s x_{T+1}$. For $t=1$, the buyer solves (3) without the constraint (4).

It should be clear that an optimal policy for problem RHF will be extremely complex and perhaps unattractive for

\footnotetext{
${ }^{1}$ Even with independent demands, there is value to RHF contracts as the commitments are influenced by the inventory position of the buyer. Our model can be extended to incorporate a dependent demand process generated via, say, a forecasting methodology. The heuristics and lower bound analyses under such an extension will remain unchanged.
} 
implementation. This motivates us to develop some heuristics. We discuss these in the next section. We close this section with a discussion of measurements for variability in the order process and accuracy of advance information it generates.

\subsection{Order Process Measurements}

An RHF contract attempts to serve two purposes. First, it restricts the variability in the order process to be less than the variability in the demand process. Second, information regarding future orders is conveyed to the supplier through commitments for future periods made by the buyer. ${ }^{2}$ Thus it is useful to measure the effectiveness of RHF contracts in achieving these two purposes. We propose two metrics.

Order Process Variability

Recall that $Q_{t, t}$ is the order quantity in period $t$ under the RHF contract. It should be clear that in a finite horizon problem, the average quantity ordered will vary depending upon the various parameters (purchase cost, flexibilities, etc.) of the contract. Therefore, we focus on the coefficient of variation $(\mathrm{CV})$ of period $t$ order, $\mathrm{ORCV}_{t}$, defined as:

$$
\mathrm{ORCV}_{t}=\frac{\sqrt{\mathbf{E}\left[Q_{t, t}^{2}\right]-\left\{\mathbf{E}\left[Q_{t, t}\right]\right\}^{2}}}{\mathbf{E}\left[Q_{t, t}\right]} .
$$

This metric captures the relative variability in the order process and can be used by a supplier for capacity investment and production planning decisions.

\section{Advance Information}

By making commitments for the future using RHF contracts a buyer also provides advance information regarding (future) orders. Naturally, a supplier would be interested in knowing how good this advance information is; for example, he may use this information to plan his procurement/production. We measure the mean absolute deviation (MAD) between the commitment made in period $t$ for period $t+i$ and the actual order placed in period $t+i .^{3}$ That is,

$$
\mathrm{MAD}_{t, t+i}=\mathbf{E}\left|Q_{t, t+i}-Q_{t+i, t+i}\right|
$$

Using computational studies we will characterize the behavior of $\mathrm{MAD}_{t, t+i}$ as $t$ approaches $t+i$ for various levels of

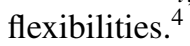

\footnotetext{
${ }^{2}$ One can also argue that another benefit of commitments is that it increases sales for the supplier by forcing the buyer to buy a minimum quantity every period. While this effect is valid for shorthorizon problems, under backlogging assumption of demand, its value diminishes as horizon gets longer.

${ }^{3}$ Observe that this is different from the usual MAD measure for a random variable where the average absolute deviation from the mean of the random variable is taken. The expression in (6) is more appropriate for the current situation.

${ }^{4}$ While MAD measures the absolute deviation, we comment on whether there is an upward or downward bias in commitments vis-a-vis orders in our numerical studies; see Section 6.
}

The validity of the MAD metric needs some justification. Clearly the supplier relies on the early commitments to plan her procurement/production. Therefore, it is important to have reliable commitments. Whether or not the MAD metric defined above is useful will then naturally depend on the supplier's planning process. An explicit model of the supplier is beyond the scope of this article. Therefore, we posit scenarios when the proposed MAD metric would be useful. Commitments by a buyer usually entail order compliance by the supplier to provide quantities up to the upper bound dictated by the level of flexibility in the contract. If the supplier has no alternate sources of supply, then given a finite production lead time he is bound to produce up to the upper limit of the commitment one-leadtime ahead of the actual order. Under such a scenario, MAD is admittedly not very useful within the production leadtime. Outside of the lead time of production, it may be used for better production or capacity planning. On the other hand, a supplier who has alternate sources of supply (e.g., flexible capacity or an outside option) may not, a priori, produce up to the upper limit of the forecast within the production leadtime. ${ }^{5}$ In such situations early information may help the supplier to better forecast the buyer's orders, produce the optimal quantities and reduce its costs.

\section{HEURISTICS AND A LOWER BOUND}

In this section, we present two heuristics to solve the general RHF contract presented earlier. We will start with an OLFC solution mechanism. We call this the OLFC-RHF heuristic. We then introduce a specialization of the RHF contract, namely the ZLF contract. We show that the optimal solution to a specific form of ZLF contract gives us an upper bound on the optimal solution of the general RHF contract. This is called the ZLF-UB heuristic. Finally, we present a lower bound for the RHF contract.

\subsection{OLFC-RHF Heuristic}

The basic idea in the OLFC approach is to determine the optimal periodical commitments at the beginning of period $t$, assuming that these commitments will not be changed at a

\footnotetext{
${ }^{5}$ For example, if the supplier has flexible capacity, she may decide to produce less than the upper limit of the forecast, take a risk, and if the actual order placed by the buyer turns out to be too large, make up the deficit using a faster but more expensive production mode. Moreover, a supplier that sells goods to several buyers may sign a flexibility contract with some of the buyers. In this case, the supplier may pool the risk over the buyers' demand. If the demand turns to be too large the buyers with a flexibilty contract will get first priority, and their demand will be satisfied. The other buyers will get only part of their orders.
} 
future period. In general OLFC policies are not optimal but are a "satisfactory mode of control for many problems" (Bertsekas [9], p. 149). We refer a problem formulation to derive the OLFC solution to the RHF contract as the OLFC-RHF problem. The optimal solution to the OLFC-RHF problem will then be a heuristic to the RHF contract. We call this the OLFC-RHF heuristic.

Let $Q_{t}(i)$ denote the sum $Q_{t, t}+\cdots+Q_{t, i}$ and $D_{t}(i)$ denote the sum $D_{t}+\cdots+D_{t+i}$. Let $J\left(Q_{t}(t), \ldots, Q_{t}(T)\right)$ be the total expected costs for periods $t$ through $T$. We write,

$$
J\left(Q_{t}(t), \ldots, Q_{t}(T)\right)=c Q_{t}(T)+\sum_{i=t}^{T} M_{t, i}\left(Q_{t}(i)\right),
$$

where

$$
\begin{aligned}
M_{t, i}\left(Q_{t}(i)\right)= & h \mathbf{E}\left[\max \left\{Q_{t}(i)-D_{t}(i), 0\right\}\right] \\
& +p \mathbf{E}\left[\max \left\{D_{t}(i)-Q_{t}(i), 0\right\}\right]
\end{aligned}
$$

is the expected holding and penalty costs in period $i<T$ for a problem starting in period $t \leq i$. For $i=T$, we write,

$$
\begin{aligned}
M_{t, T}\left(Q_{t}(T)\right)=(h-s) \mathbf{E}[ & \left.\max \left\{Q_{t}(T)-D_{t}(T), 0\right\}\right] \\
& +p \mathbf{E}\left[\max \left\{D_{t}(T)-Q_{t}(T), 0\right\}\right]
\end{aligned}
$$

Let $Q_{1}(0)=0$. The OLFC-RHF problem is defined as follows: For period one:

$$
\begin{aligned}
\min _{Q_{1}(1), \ldots, Q_{1}(T)} J\left(Q_{1}(1), \ldots, Q_{1}(T)\right) \\
\text { s.t. } \quad Q_{1}(i) \geq Q_{1}(i-1) \quad \forall i=1, \ldots, T .
\end{aligned}
$$

Constraints (8) ensure that the commitment in every period is non-negative. For period $t=2, \ldots, T$

$$
\begin{aligned}
& \min _{Q_{t}(t), \ldots, Q_{t}(T)} J\left(x_{t}+Q_{t}(t), \ldots, x_{t}+Q_{t}(T)\right) \\
& \text { subject to } \\
& Q_{t}(t+i) \geq Q_{t}(t+i-1) \quad \forall i=0, \ldots, T-t \\
& \left(1-\underline{\alpha}_{t, t+i}\right) Q_{t-1, t+i} \leq Q_{t, t+i} \leq\left(1+\bar{\alpha}_{t, t+i}\right) Q_{t-1, t+i} \\
& \forall i=0, \ldots, T-t .
\end{aligned}
$$

We first present the solution for period one. For convenience, we refer to the first period problem as the static problem because flexibility plays no role in its solution. It is easy to see that the static problem is a convex problem in $Q_{1}(1), \ldots, Q_{1}(T)$. Let $S_{i}, i=1, \ldots T-1$ solve

$$
F_{1, i}\left(S_{i}\right)=\frac{p}{p+h},
$$

and $S_{T}$ solve:

$$
F_{1, T}\left(S_{T}\right)=\frac{p-c}{p+(h-s)},
$$

where $F_{1, i}$ is the cumulative distribution function of $D_{1, i}$ for $i=1, \ldots, T$. Observe that $S_{1} \leq S_{2} \leq \cdots \leq S_{T-1}$. The following Lemma gives the structure of the optimal policy for the static problem.

\section{LEMMA 4.1: Let $S_{0} \equiv 0$.}

1. If $S_{T} \geq S_{T-1}$ then the optimal solution is $Q_{1, i}^{*}=$ $S_{i}-S_{i-1}$ for $t=1, \ldots, T$.

2. If $S_{T}<S_{T-1}$ then there exists a $k^{*}<T$ and $\tilde{S}_{k^{*}}$, such that

$$
Q_{1, i}^{*}=\left\{\begin{array}{ll}
S_{i}-S_{i-1} & \text { if } i<k^{*} \\
\tilde{S}_{k^{*}}-S_{i} & \text { if } i=k^{*} \\
0 & \text { if } i>k^{*}
\end{array} .\right.
$$

Algorithm 1 described in Appendix A results in the optimal solution for period one of the OLFC-RHF problem. Convexity of the cost function $J(\cdot)$ guarantees that the sum of first partials of $J(\cdot)$ in step 2 of Algorithm 1 are monotone and thus the optimum can be found by a simple binary search. We now illustrate the structure of the optimal solution for the static problem when demands are stationary and follow a Normal distribution with mean $\mu$ and standard deviation $\sigma$. We have:

$$
\begin{aligned}
S_{i} & =i \mu+k \sqrt{i} \sigma, \quad i=1, \ldots T-1, \quad \text { and } \\
S_{T} & =T \mu+k_{T} \sqrt{T} \sigma,
\end{aligned}
$$

where $\Phi(k)=\frac{p}{p+h}$ and $\Phi\left(k_{T}\right)=\frac{p-c}{p+(h-s)}$ and $\Phi(\cdot)$ is the cumulative standard normal density. In addition, if conditions of part 1 of Lemma 4.1 are satisfied, we have

$$
Q_{1, i}^{*}=S_{i}-S_{i-1}=\mu+k(\sqrt{i}-\sqrt{i-1}) \sigma, \quad i=1, \ldots, T-1,
$$

and

$$
\begin{aligned}
Q_{1, T}^{*} & =S_{T}-S_{T-1} \\
& =\mu+\left(k_{T} \sqrt{T}-k \sqrt{T-1}\right) \sigma .
\end{aligned}
$$

Observe that the periodical commitments are decreasing with time (converging towards the mean demand), except for the last period of non-zero commitment.

We now solve the OLFC-RHF problem for period $t>1$. The solution to the problem in period $t>1$ can be obtained by solving a relaxed problem obtained by ignoring constraints (11). Subsequently, we check if the constraints are satisfied. If they are not, then we simply purchase quantities as specified by the constraints and carry forward a surplus or deficit (between the unconstrained quantity and the upper or lower bound) to the next period. This procedure is presented as Algorithm 2 in Appendix A. 


\subsection{ZLF-UB Heuristic}

We now define the following specialization of the RHF contract. In period one the buyer makes a commitment to purchase certain quantities in each period of the horizon. In any given period $t$, he is allowed to adjust his actual order quantity, $Q_{t, t}$ within some prespecified limits; however, the buyer is not allowed to update the commitments for future periods. Because the buyer is only allowed to adjust the order quantity in the current period, we call this a ZLF contract. Thus a ZLF contract is a special case of the RHF contract with $\underline{\alpha}_{t, i}=0$ and $\bar{\alpha}_{t, i}=0$ for $t>i$. We will show that the optimal solution to an appropriately defined ZLF contract gives us an upper bound to the optimal solution of the corresponding RHF contract. We postpone the discussion of the optimal policy structure for the generic ZLF contract and a solution procedure to Section 5 .

Consider the RHF contract with flexibilities $[\alpha]$ and $[\bar{\alpha}]$. Define a flexibility matrix $[\underline{\beta}]$ such that $\underline{\beta}_{i, j}=0$ for $i \neq j$ and $\underline{\beta}_{i, i}=\underline{\alpha}_{i, i}$. Similarly, define another flexibility matrix $[\bar{\beta}]$ such that $\bar{\beta}_{i, j}=0$ for $i \neq j$ and $\bar{\beta}_{i, i}=\bar{\alpha}_{i, i}$. Clearly, a contract with flexibility matrices $[\beta]$ and $[\bar{\beta}]$ is a ZLF contract. We will henceforth refer to it as ZLF-UB contract. Thus using a ZLF-UB contract, the buyer initially gives a commitment $Q_{1,1}, \ldots, Q_{1, T}$ for the entire horizon. Subsequently, in any period $t$ he adjusts his orders $Q_{t, t}$ such that,

$$
\left(1-\underline{\beta}_{t, t}\right) Q_{1, t} \leq Q_{t, t} \leq\left(1+\bar{\beta}_{t, t}\right) Q_{1, t} .
$$

Furthermore, he does not update any commitments for the future. Because the ZLF-UB contract is a restriction on the RHF contract, we have the following result.

PROPOSITION 4.1: At optimality the expected cost of the ZLF-UB contract gives an upper bound on the expected costs of a RHF contract.

We refer to the optimal solution of the ZLF-UB contract as a ZLF-UB heuristic for the RHF contract.

To summarize, we have presented two heuristics for the solution of a general RHF contract. The first one, called OLFC-RHF, is an open loop feedback control (OLFC) solution implemented in a rolling horizon manner. Next, the ZLF-UB heuristic is an optimal solution to a ZLF-UB contract, which is a restriction on the original RHF contract. It is not a priori clear which of the two heuristics-OLFCRHF or ZLF-UB - will perform better. We report numerical comparisons between the two in section 6 .

\subsection{A Lower Bound}

An obvious lower bound is given by solving the finite horizon newsvendor problem. Using computational studies (discussed later), however, we observe that it is not a good lower bound. We now derive another lower bound, which we numerically demonstrate to be tight. Consider the following specialization of the RHF contract with flexibilities $[\underline{\alpha}]$ and $[\bar{\alpha}]$. Define matrices $[\gamma]$ and $[\bar{\gamma}]$ such that,

$$
\underline{\gamma}_{i, j}= \begin{cases}0 & \text { if } i \neq j \\ 1-\prod_{k=1}^{j}\left(1-\underline{\alpha}_{k, j}\right) & \text { if } i=j\end{cases}
$$

and,

$$
\bar{\gamma}_{i, j}= \begin{cases}0 & \text { if } i \neq j \\ \prod_{k=1}^{j}\left(1+\bar{\alpha}_{k, j}\right)-1 & \text { if } i=j .\end{cases}
$$

A contract with flexibilities $[\gamma]$ and $[\bar{\gamma}]$ is a ZLF contract introduced in the previous section. Since we will shortly demonstrate that this specific ZLF contract gives a lower bound to the original RHF contract, we will henceforth refer to it as a ZLF-LB contract.

PROPOSITION 4.2: The optimal solution to the ZLF-LB contract gives a lower bound to the optimal solution to the RHF contract.

\section{THE ZLF CONTRACT: OPTIMAL POLICY AND ALGORITHM}

We now derive the optimal policy structure for a ZLF contract and also outline a solution procedure. The buyer's objectives are to determine: (a) ex ante the optimal commitments for every period given the flexibility parameters, and (b) for the chosen commitments, the optimal (dynamic) order policy for every period. This can be modeled as a two-stage problem. We first show that the optimal (dynamic) order policy given a vector of commitments and flexibilities is a modified base stock policy. Subsequently, we present an iterative procedure to compute (a) and (b) above, as well as demonstrate its convergence.

\subsection{Optimal Adjustment Policy Given Commitments}

Consider a ZLF contract with flexibility matrices $[\underline{\alpha}]$ and $[\bar{\alpha}]$. We first analyze part (b) above to determine the optimal order policy given a vector of commitments, $Q_{1,1}, \ldots, Q_{1, T}$, made in period 1 for periods 1 through $T$. Recall that $Q_{1, i}$ is the commitment in period 1 for period $i$. The order quantity in period $i, Q_{i, i}$, must satisfy the flexibility constraint $\left(1-\underline{\alpha}_{i, i}\right) Q_{1, i} \leq Q_{i, i} \leq\left(1+\bar{\alpha}_{i, i}\right) Q_{1, i}$.

Let the expected cost from period $i$ through $T$ given a starting inventory of $x_{i}$ and commitments $Q_{1, i}, \ldots, Q_{1, T}$ for periods $i$ through $T$ made in period 1 be

$$
\begin{aligned}
A C_{i}\left(Q_{i, i} \mid x_{i}, Q_{1, i}, \ldots, Q_{1, T}\right)=c Q_{1, i}+L_{i}\left(x_{i}+Q_{i, i}\right) \\
+\mathbf{E}_{D_{i+1}}\left\{A C_{i+1}^{*}\left(x_{i+1}, Q_{1, i+1}, \ldots, Q_{1, T}\right)\right\}
\end{aligned}
$$


where $x_{i+1}=x_{i}+Q_{i, i}-D_{i}$ for $i=1, \ldots, T$ is the inventory balance equation. Define $A C_{T+1}^{*}\left(x_{T+1}\right) \equiv-s x_{T+1}$. The optimal order policy for period $i, i=2, \ldots, T$, can then be derived by solving the following dynamic programming formulation:

$$
\begin{aligned}
& A C_{i}^{*}\left(x_{i}, Q_{1, i}, \ldots, Q_{1, T}\right) \\
& =\min _{Q_{i, i}} A C_{i}\left(Q_{i, i} \mid x_{i}, Q_{1, i}, \ldots, Q_{1, T}\right) \\
& \quad \text { subject to } \\
& \quad\left(1-\underline{\alpha}_{i, i}\right) Q_{1, i} \leq Q_{i, i} \leq\left(1+\bar{\alpha}_{i, i}\right) Q_{1, i}
\end{aligned}
$$

For $i=1$ we solve (17) without the constraint (18). The cost function of Problem (P2) is identical to the cost function of a newsvendor problem, which is known to be convex. It is easy to see that the feasible set of the problem (P2) is convex. Thus,

\section{LEMMA 5.1: Problem (P2) is convex.}

Given a commitment vector $Q_{1,1}, \ldots, Q_{1, T}$, the optimal order policy for period $i$ is given by the following proposition.

PROPOSITION 5.1: There exists $Z_{i}$ for $i=1, \ldots, T$ such that the optimal order policy for period 1 is

$$
Q_{1,1}^{*}=\max \left\{Z_{1}-x_{1}, 0\right\},
$$

and for periods $i=2, \ldots, T$ is:

$$
Q_{i, i}^{*}=\left\{\begin{array}{lll}
\left(1-\underline{\alpha}_{i, i}\right) Q_{1, i} & \text { if } & Z_{i}-x_{i} \leq\left(1-\underline{\alpha}_{i, i}\right) Q_{1, i} \\
Z_{i}-x_{i} & \text { if } & \left(1-\underline{\alpha}_{i, i}\right) Q_{1, i}<Z_{i}-x_{i} \\
& & \leq\left(1+\bar{\alpha}_{i, i}\right) Q_{1, i} \\
\left(1+\bar{\alpha}_{i, i}\right) Q_{1, i} & \text { if } & Z_{i}-x_{i}>\left(1+\bar{\alpha}_{i, i}\right) Q_{1, i}
\end{array}\right.
$$

The $Z_{i}$ 's are similar to the modified base stock levels described in Federgruen and Zipkin [13] for the capacitated newsvendor problem. The following problem (ZLF) then solves for optimal commitments $Q_{1,1}, \ldots, Q_{1, T}$.

$$
\text { (ZLF) } \min _{Q_{1,1}, \ldots, Q_{1, T}} A C_{1}^{*}\left(x_{1}, Q_{1,1}, \ldots, Q_{1, T}\right)
$$

LEMMA 5.2: The function $A C_{1}^{*}\left(x_{1}, Q_{1,1}, \ldots, Q_{1, T}\right)$ is convex in $\left(x_{1}, Q_{1,1}, \ldots, Q_{1, T}\right)$.

Observe that the solution to (ZLF) is a set of $T$ periodical commitments, $Q_{1,1}^{*}, \ldots, Q_{1, T}^{*}$. From an operations point of view it is also important to know the optimal modified base stock levels, $Z_{1}^{*}, \ldots, Z_{T}^{*}$, because they will determine the actual order quantity for a period given by (19). Convexity of $A C_{1}^{*}\left(x_{1}, Q_{1,1}, \ldots, Q_{1, T}\right)$ together with the facts that

$$
\lim _{Q_{1,1}, \ldots, Q_{1, T} \rightarrow \infty} A C_{1}^{*}\left(x_{1}, Q_{1,1}, \ldots, Q_{1, T}\right) \rightarrow \infty
$$

and $Q_{1, i} \geq 0$, for $i=1, \ldots, T$ ensure that $A C_{1}^{*}\left(x_{1}, Q_{1,1}, \ldots\right.$, $Q_{1, T}$ ) has a global minimum. However, a direct solution to the (ZLF) problem is extremely difficult. We now outline an iterative procedure to obtain the optimal commitments for the ZLF problem.

\subsection{Algorithm for Optimal Commitments}

Thus far we have shown that for a given commitment vector, Problem (P2) gives the optimal order policy defined by the modified base stock levels $Z_{1}, \ldots, Z_{T}$. Observe that $Z_{1}, \ldots, Z_{T}$ are implicitly functions of $Q_{1,1}, \ldots, Q_{1, T}$. An iterative approach to solve for the optimal commitments given by (ZLF) will involve repeatedly executing the following steps (the superscript denotes iteration index): At iteration $j$

Step (a) For a given commit vector $Q_{1,1}^{j-1}, \ldots, Q_{1, T}^{j-1}$, solve (P2) to get the base stock policy $Z_{1}^{j}, \ldots, Z_{T}^{j}$, and

Step (b) Given $Z_{1}^{j}, \ldots, Z_{T}^{j}$, get a new commit vector $Q_{1,1}^{j}, \ldots, Q_{1, T}^{j}$

until some convergence criteria is satisfied. We now outline a procedure to update the commitment vector-Step (b) above-for a given base stock policy.

Updating the Commitment Vector

Recall that the actual purchase quantity in a period $i$ is given by (19), with appropriate superscript for iteration number. Define $B C_{i}^{j}\left(x_{i}, Q_{1,1}^{j-1}, \ldots, Q_{1, i-1}^{j-1}, Q_{1, i}^{j}, \ldots, Q_{1, T}^{j} \mid\right.$ $\left.Z_{1}^{j}, \ldots, Z_{T}^{j}\right)$ as the expected cost starting at period $i$ through period $T$, for an initial inventory level of $x_{i}$, and commitment vector $\left(Q_{1,1}^{j-1}, \ldots, Q_{1, i-1}^{j-1}, Q_{1, i}^{j}, \ldots, Q_{1, T}^{j}\right)$, and given the modified base stock levels $Z_{1}^{j}, \ldots, Z_{T}^{j}$ which are functions of $\left(Q_{1,1}^{j-1}, \ldots, \ldots, Q_{1, T}^{j-1}\right)$. It is important to note that in order to evaluate the function $B C_{i}^{j}(\cdot \cdot \cdot)$ we need to know the value of the initial inventory $x_{i}$ in period $i$. But $x_{i}$ is a random variable whose probability measure is not known and unlikely to have a simple structure. Therefore, we evaluate the function using simulation, the exact details of which are described in Appendix C. Then:

$$
\begin{array}{r}
B C_{i}^{j}\left(x_{i}, Q_{1,1}^{j-1}, \ldots, Q_{1, i-1}^{j-1}, Q_{1, i}^{j}, \ldots, Q_{1, T}^{j} \mid Z_{1}^{j}, \ldots, Z_{T}^{j}\right)= \\
c Q_{i, i}^{j}+L_{i}\left(x_{i}+Q_{i, i}^{j}\right)+\mathbf{E}_{D_{i}}\left\{B C _ { i + 1 } ^ { j } \left(x_{i+1}, Q_{1,1}^{j-1}, \ldots,\right.\right. \\
\left.\left.Q_{1, i-1}^{j-1}, Q_{1, i}^{j}, \ldots, Q_{1, T}^{j} \mid Z_{1}^{j}, \ldots, Z_{T}^{j}\right)\right\}
\end{array}
$$


where $Q_{i, i}^{j}$ is given by Proposition 5.1 and $x_{i+1}=x_{i}+Q_{i, i}^{j}-$ $D_{i}$.

We now define problem (P3) as follows:

$$
\begin{gathered}
\min _{Q} B C_{i}^{j}\left(x_{i}, Q_{1,1}^{j-1}, \ldots, Q_{1, i-1}^{j-1}, Q, Q_{1, i+1}^{j}, \ldots,\right. \\
\left.Q_{1, T}^{j} \mid Z_{1}^{j}, \ldots, Z_{T}^{j}\right) \quad \text { for } i=T, \ldots, 1
\end{gathered}
$$

and $Q_{1, i}^{j}=\operatorname{argmax}_{Q} B C_{i}^{j}\left(x_{i}, Q_{1,1}^{j-1}, \ldots, Q_{1, i-1}^{j-1}, Q, Q_{1, i+1}^{j}\right.$, $\left.\ldots, Q_{1, T}^{j} \mid Z_{1}^{j}, \ldots, Z_{T}^{j}\right)$.

To solve problem (P3) at iteration $j$ it is necessary to solve $T$ one-dimensional problems beginning with the last period to obtain $Q_{1, T}^{j}$ and sequentially solving for $Q_{1, T-1}^{j}, \ldots, Q_{1,1}^{j}$.

Observe that in solving problem (P3) we use the modified base stock levels $\left(Z_{1}^{j}, \ldots, Z_{T}^{j}\right)$ that were obtained in the solution of (P2) (at iteration $\mathrm{j}$ ). In addition, the new commitment $Q_{1, i}^{j}$ is calculated assuming the commitments $Q_{1,1}^{j-1}, \ldots, Q_{1, i-1}^{j-1}$ that were determined in the previous iteration. Thus, in general, the commitments obtained by solving problem (P3) are not optimal for ZLF. However, the commitment obtained by solving problem (P3) will always reduce the total cost of ZLF. Algorithm 3 in Appendix A essentially solves problems (P2) and (P3) iteratively to obtain the optimal solution for ZLF. Observe that the modified base stock levels uniquely determine the optimal order quantity and thus problem (P2) can be defined as a problem in the space of $Z_{i}$ instead of $Q_{i, i}$.

For an initial solution to the commitment vector we use the solution to the static problem. Clearly when the magnitude of the flexibility is small, optimal commitments for the static problem are close to optimal commitments for the ZLF problem. As the magnitude of flexibility increases our computational studies indicate that the ZLF solution differs from the static solution only in last few periods. Proposition 5.2 guarantees the convergence of Algorithm 3.

PROPOSITION 5.2: Algorithm 3 converges to the optimal solution of problem (ZLF).

\section{COMPUTATIONAL STUDIES}

We now describe numerical studies to study the behavior of RHF contracts. The parameters of the contract include a purchase price, flexibilities, a decision horizon, and a demand pattern. First we show effectiveness of the approximations developed in this article by comparing the performance of various heuristics against the lower bound. Subsequently, we perform sensitivity analysis to gain insights into the problem. From a managerial perspective, we are primarily interested in answers to the following types of questions: (i) How much flexibility of any given type is sufficient? (ii) What is the value of an additional (say, 5\%) flexibility? (iii) What is the impact of various types of contracts on the order process variability? (iv) What is the nature of advance information provided by an RHF contract? In addition, using the OLFC-RHF and ZLF-UB heuristics, we comment on the appropriateness of a general RHF contract versus a suitably defined ZLF contract from the perspective of both the supplier and the buyer. Clearly, answers to such questions may depend on the demand and cost parameters. While we have attempted an extensive computational study, it is by no means exhaustive.

The following parameters are used for our computations. The horizon length is 12 periods. Demands are assumed to either (i) STATIONARY with a mean of 100.0 per period, or (ii) non-stationary with four possible patterns of the mean, viz., DECREASING (starting from 155.0 down to 100.0 in steps of 5.0), INCREASING (starting from 100.0 to 155.0 in steps of 5.0), TRIANGULAR (starting from 100.0, increasing to 130.0 and then decreasing to 105.0 all in steps of 5.0), and WEDGE shaped (starting from 130.0 decreasing to 100.0 and then increasing back to 125.0 in steps of 5.0). The coefficient of variation $(\mathrm{CV})$ of demand (for both stationary and non-stationary patterns) was chosen from $\{0.25,0.33,0.5\}$. We keep the CV of demand stationary even as mean demand values change. The per unit purchase price was $\$ 5.0$ and the per unit holding cost was $\$ 0.1$. The per unit penalty cost was chosen from $\{\$ 7.5,10.0,15.0,25.0\}$. The end-of-horizon salvage values were chosen to be either equal to the purchase cost (at \$5.0) or $20 \%$ of purchase cost (at \$1.0). In the RHF contracts considered for computational purposes, we assumed that the flexibility available was stationary and symmetric (the heuristics can be used even when flexibility is non-stationary and asymmetric). That is, $\bar{\alpha}_{t, t+i}$ were identical for all $t \in[1, T]$ and $i \in[0, T-t]$ (stationary) and $\underline{\alpha}_{t, t+i}=\bar{\alpha}_{t, t+i}$ (symmetric). So henceforth when we say $5 \%$ flexibility we imply that $\bar{\alpha}_{t, t+i}=\underline{\alpha}_{t, t+i}=0.05$ for all $t \in[1, T]$ and $i \in[0, T-t]$. It is important to notice that even when flexibilities are stationary, due to the cumulative effect (through ability to change future orders) the "effective" flexibilities are non-stationary. More specifically, the "effective" flexibility is smaller for a shorter time horizon and larger for a longer time horizon.

Demands were assumed to follow a normal density function, truncated at zero. Before we present our sensitivity analysis results, we briefly outline a few details regarding our computational procedures. Implementation of algorithms 1 and 2 require computation of first partials of the expected cost function; we use Monte-Carlo methods for the same. The demand points were generated using the polar method of normal deviates due to G.E.P Box, M.E. Muller, and G. Marsaglia [See Knuth [14], page 117]. For each period, the number of demand points generated with the underlying normal distribution was 2500 . To get confidence intervals, we repeated each computation 10 times; the coefficient of variation of the average costs was less than $0.25 \%$. In the sequel, 

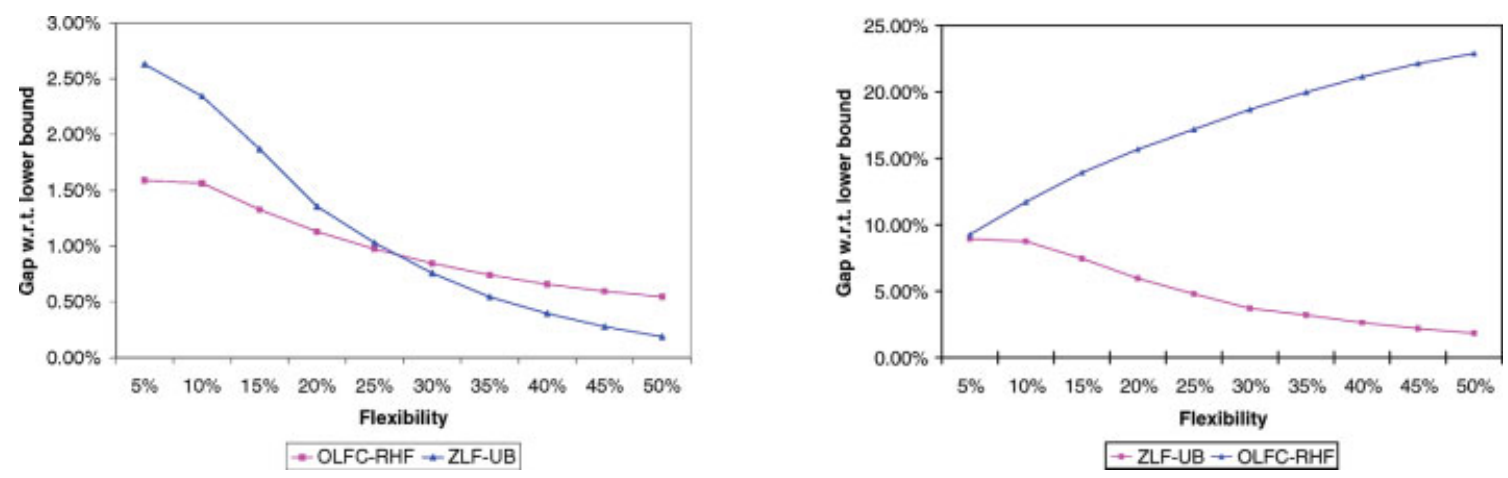

Figure 2. OLFC-RHF versus ZLF-UB heuristic for STATIONARY demand with CV $=0.5$ and salvage value $=100 \%$ and $20 \%$ of purchase cost respectively. [Color figures can be viewed in the online issue, which is available at www.interscience.wiley.com.]

to avoid clutter, we do not report the details of the confidence intervals. Further details regarding the implementation of the heuristics and the lower bound are given in Appendix C.

We now discuss the performance of the heuristics, some comparisons, sensitivity analysis, and insights gained.

\subsection{Performance of the Heuristics}

\subsubsection{Comparison of Heuristics}

We compared the performance of OLFC-RHF and ZLFUB heuristics for various values of flexibilities. The percentage gap between the two heuristics with respect to the lower bound was compared for both high and low salvage values. The results for stationary demand with a $\mathrm{CV}=0.5$ are plotted in Fig. 2. Similar results are observed for the four nonstationary demand patterns. For brevity of exposition, we do not report the comparative results between the heuristics for the various nonstationary patterns. As we see from Fig. 2, when salvage value is high (left graph) the OLFC-RHF heuristic outperforms the ZLF-UB heuristic for lower values of flexibility. We see a similar behavior for lower values of the $\mathrm{CV}$ of demand. An intuitive argument for the observed behavior is as follows: in comparison to the ZLF-UB heuristic, the rolling horizon nature of the OLFC-RHF heuristic, through its ability to adjust future commitments, provides a larger flexibility to adjust orders. However, when the original RHF contract itself offers high flexibilities, this advantage diminishes and since ZLF-UB solves for the optimal policy whereas OLFC-RHF uses a suboptimal policy, the former dominates.

In contrast, as we see from Fig. 2 (right half), the ZLFUB heuristic outperforms the OLFC-RHF heuristic for lower salvage values. In addition, performance of the OLFC-RHF heuristic degrades as flexibility increases, which is counterintuitive. The pattern repeats at lower values of the $\mathrm{CV}$ of demand. In this example when salvage values are low, the initial commitment developed using the OLFC-RHF heuristic for the last few periods of the planning horizon are zero. In periods with non-zero commitments the OLFC-RHF heuristic has the ability to order smaller quantities as flexibilities increase. This turns out to be a disadvantage when entering the last few periods of zero commitments. This phenomenon occurs since an OLFC-RHF heuristic, being open-loop, does not consider the future (decisions or possible states of nature) in its decision making. We observed that the OLFCRHF heuristic outperforms the ZLF-UB heuristic in the first 10 (of the 12) periods in the horizon. However, it incurs huge shortages in the last two periods due to its inability to order non-zero quantities in the last two periods (since it's initial commitments for these periods were zero). Over the planning horizon OLFC-RHF performs worse than ZLF-UB since the penalty cost is an order of magnitude greater than the holding cost. The ZLF-UB heuristic being closed-loop adjusts the commitments in the last few periods to be non-zero and avoids this problem.

To summarize, we observe that for the various demand patterns tested the OLFC-RHF heuristic performs better than the ZLF-UB (at least for low to medium levels of flexibility) when salvage values are high. The gap between the two, however, is small. On the other hand, when salvage values are low, ZLF-UB appears to perform better than the OLFC-RHF and the gap between the two is larger.

\subsubsection{Performance of the Heuristics}

On the basis of these observations, we compared the performance of the OLFC-RHF heuristic (for high salvage value) and of the ZLF-UB heuristic (for low salvage value) to the lower bound (ZLF-LB) for various levels of the coefficient of variation $(\mathrm{CV})$ for each demand pattern. We summarize the key observations here; detailed results are available in Bassok and Anupindi [7]. We observe that the OLFC-RHF 
Table 1. Performance of the OLFC-RHF and ZLF-UB heuristics w.r.t. the newsvendor problem for STATIONARY demand.

\begin{tabular}{|c|c|c|c|c|c|c|c|c|c|c|c|c|}
\hline \multirow[b]{3}{*}{ Flexibility $\%$} & \multicolumn{6}{|c|}{ OLFC-RHF Heuristic } & \multicolumn{6}{|c|}{ ZLF-UB Heuristic } \\
\hline & \multicolumn{2}{|c|}{$\mathrm{CV}=0.25$} & \multicolumn{2}{|c|}{$\mathrm{CV}=0.33$} & \multicolumn{2}{|c|}{$\mathrm{CV}=0.50$} & \multicolumn{2}{|c|}{$\mathrm{CV}=0.25$} & \multicolumn{2}{|c|}{$\mathrm{CV}=0.33$} & \multicolumn{2}{|c|}{$\mathrm{CV}=0.50$} \\
\hline & Gap \% & ORCV & Gap \% & ORCV & Gap \% & ORCV & Gap \% & ORCV & Gap \% & ORCV & Gap \% & ORCV \\
\hline 0 & 2.07 & - & 2.68 & - & 3.97 & - & 2.07 & - & 2.68 & - & 3.97 & - \\
\hline 5 & 1.23 & 0.11 & 1.58 & 0.12 & 2.46 & 0.13 & 1.36 & 0.03 & 1.97 & 0.03 & 3.51 & 0.04 \\
\hline 10 & 0.91 & 0.15 & 1.14 & 0.17 & 1.78 & 0.20 & 0.87 & 0.08 & 1.37 & 0.07 & 2.56 & 0.07 \\
\hline 15 & 0.75 & 0.17 & 0.90 & 0.20 & 1.40 & 0.24 & 0.56 & 0.12 & 1.00 & 0.12 & 1.94 & 0.10 \\
\hline 20 & 0.66 & 0.19 & 0.75 & 0.22 & 1.14 & 0.28 & 0.38 & 0.16 & 0.73 & 0.16 & 1.37 & 0.14 \\
\hline 25 & 0.60 & 0.20 & 0.65 & 0.24 & 0.97 & 0.30 & 0.32 & 0.19 & 0.57 & 0.2 & 1.02 & 0.18 \\
\hline 30 & 0.55 & 0.22 & 0.58 & 0.26 & 0.83 & 0.33 & 0.30 & 0.21 & 0.52 & 0.23 & 0.74 & 0.22 \\
\hline 35 & 0.53 & 0.23 & 0.53 & 0.27 & 0.72 & 0.35 & 0.46 & 0.22 & 0.48 & 0.26 & 0.52 & 0.27 \\
\hline 40 & 0.51 & 0.24 & 0.49 & 0.29 & 0.63 & 0.37 & 0.41 & 0.24 & 0.55 & 0.28 & 0.37 & 0.31 \\
\hline 45 & 0.50 & 0.25 & 0.46 & 0.30 & 0.56 & 0.39 & 0.50 & 0.24 & 0.67 & 0.3 & 0.25 & 0.34 \\
\hline 50 & 0.49 & 0.25 & 0.44 & 0.32 & 0.52 & 0.41 & 0.63 & 0.24 & 0.83 & 0.31 & 0.16 & 0.38 \\
\hline
\end{tabular}

heuristic performs quite well relative to the lower bound for a wide range of $\mathrm{CV}$ of demand and across all demand patterns as a function flexibilities. The average gaps for salvage value equal $100 \%$ and $20 \%$ of purchase cost, respectively, are $0.76 \%$ and $5.17 \%$; the respective maximum gaps are $1.69 \%$ and $10.9 \%$. We also observe that for high salvage values, the gap between the best heuristic (OLFC-RHF) and the lower bound is quite small whereas for low salvage values the gap between the best heuristic (ZLF-UB) and the lower bound is somewhat larger.

For most mature product lines that are not towards the end of their product life cycles, it is reasonable to assume that the salvage value of the product at the end of the horizon will be its purchase cost. On the other hand for products with a short lifecycle, the salvage value could be significantly less than the purchase cost. In the sequel, for brevity of exposition, we assume salvage value equal to purchase cost. The nature of the insights we obtain carry over to other cases as well.

\subsection{Sensitivity Analysis and Insights}

\subsubsection{Flexibility versus Order Process Variability}

Recall that the base case for comparison is the unconstrained newsvendor problem that provides "unlimited" flexibility for a buyer. An RHF contract with limited flexibility clearly raises the costs to the buyer. On the other hand, as we motivated earlier, RHF contracts reduce the varibility in the order process, a potential benefit to the supplier. Using our computational studies, we first quantify the impact of flexibility on expected costs to the buyer. Simultaneously we measure the order process variability (ORCV), as defined in (5), to highlight the potential benefit to a supplier. In Table 1 , we display the percentage gap in expected cost between the newsvendor problem and the OLFC-RHF and ZLF-UB heuristics for various levels of flexibility for a stationary demand pattern. We also show ORCV for each of these levels of flexibility. ${ }^{6}$ As expected the gap between the expected cost of the two heuristics and the newsvendor problem decreases as flexibility increases. More importantly, only low to moderate levels of flexibility are required to get the expected cost within, say $1.0 \%$, of the newsvendor solution. The corresponding ORCV is quite low. For example, using OLFC-RHF with demand $\mathrm{CV}=0.33$, less than $15 \%$ flexibility is required to be within $1 \%$ of the corresponding newsvendor solution; in Table 1 the two numbers in bold face bracket the flexibility level for which the gap is just above and below the $1 \%$ level. At $15 \%$ flexibility, ORCV is 0.20 , which is significantly lower than the corresponding ORCV of 0.33 (equal to the demand cv) using the newsvendor model. In addition, we observe that for the same flexibility level ORCV with ZLF-UB heuristic is smaller than ORCV with OLFC-RHF heuristic. For example, for a $10 \%$ flexibility and demand $\mathrm{CV}=0.25, \mathrm{ORCV}=$ 0.15 for OLFC-RHF and 0.08 for ZLF-UB. This observation plays an important role in determining whether an RHF or ZLF contract is superior. We postpone this discussion to the end of this section.

We observe similar behavior for various nonstationary demand patterns. For brevity we only report results for the TRIANGULAR demand with $\mathrm{CV}=0.33$ in Table 2 which displays the percentage gap between the newsvendor problem and the OLFC-RHF and the ZLF-UB heuristics as well as the range of ORCV. We observe that with nonstationary demand patterns, the ORCV is also nonstationary. In Table 2 we simply report the range of ORCV giving its minimum and

\footnotetext{
${ }^{6}$ Observe that in a finite horizon model, the $\mathrm{CV}$ of orders in the early periods will be small. This is basically due to the fact the "effective" flexibility in the earlier periods is small. In the computational studies with stationary demand we observe that the CV of orders converge as the horizon length increases. We therefore compare this converged value of the $\mathrm{CV}$ of orders to the $\mathrm{CV}$ of demand and show that the $\mathrm{CV}$ of orders in a RHF contract is significantly lower than the $\mathrm{CV}$ of demand.
} 
Table 2. Performance of the OLFC-RHF and ZLF-UB heuristics w.r.t. the newsvendor problem for TRIANGULAR demand with $\mathrm{CV}=0.33$

\begin{tabular}{lccccc}
\hline & \multicolumn{2}{c}{ OLFC-RHF Heuristic } & & \multicolumn{2}{c}{ ZLF-UB } \\
\cline { 2 - 3 } \cline { 5 - 6 } Flexibility \% & Gap \% & ORCV Range & & Gap \% & ORCV Range \\
\hline 5 & 1.58 & $0.04-0.14$ & & 1.92 & $0.03-0.04$ \\
10 & $\mathbf{1 . 1 4}$ & $0.07-0.19$ & & $\mathbf{1 . 3 4}$ & $0.06-0.08$ \\
15 & $\mathbf{0 . 9 1}$ & $0.10-0.21$ & & $\mathbf{0 . 9 4}$ & $0.08-0.12$ \\
20 & 0.76 & $0.13-0.23$ & & 0.68 & $0.08-0.16$ \\
25 & 0.66 & $0.16-0.25$ & & 0.53 & $0.06-0.20$ \\
30 & 0.59 & $0.19-0.26$ & & 0.44 & $0.05-0.24$ \\
35 & 0.54 & $0.21-0.28$ & & 0.60 & $0.12-0.27$ \\
40 & 0.50 & $0.23-0.29$ & & 0.47 & $0.01-0.29$ \\
45 & 0.48 & $0.25-0.30$ & & 0.54 & $0.00-0.32$ \\
50 & 0.46 & $0.26-0.31$ & & 0.65 & $0.00-0.36$ \\
\hline
\end{tabular}

maximum across periods (excluding the first period which will have an ORCV $=0.0$ ). It is a priori unclear if the ORCV of one heuristic dominates the other.

Finally, from Table 1 we see that the expected costs are affected by both the $\mathrm{CV}$ of demand and the flexibility in the supply contract. ${ }^{7}$ Internally, it points to the value of coordination between the marketing (responsible for demand forecasting and thus affecting CV of demand) and purchasing functions (responsible for negotiating supply contracts).

\subsubsection{Decreasing returns to additional flexibility}

In Table 3, we plot the percentage decrease in total expected costs for an additional $5 \%$ flexibility for various values of the $\mathrm{CV}$ of stationary demand and observe that the marginal returns for an additional 5\% flexibility decreases drastically. Similar results are observed (not reported here) for other nonstationary patterns. Such a characterization is useful for a buyer because it allows him to consider his "willingness-topay" for additional flexibility from a supplier. Suppose the supply contract is structured as a non-linear contract (fixed fee + proportional costs) with fixed fee component a function of the flexibility. The buyer should then be willing to pay a fixed fee up to the value of additional 5\% flexibility (Table 3 ). On the other hand, a supplier may likely offer a menu of (linear price, flexibility) contracts [1]. Since our computation methods are fast, such menus can be easily evaluated.

\subsubsection{Effect of penalty costs}

To test for the effect of penalty costs we measured the average fill-rate (fraction of demand satisfied from stock) as a function of flexibility for various values of the penalty cost parameter. The results for stationary demands are graphed in

\footnotetext{
${ }^{7}$ We observe that the same is true for the non-stationary demand patterns; for brevity we do not report detailed results here.
}

Fig. 3. We observe that increased flexibility allows the buyer to offer better customer service as measured by the average fill-rate. Thus, we illustrate a link between a service measure offered to a consumer (a marketing function) with the capability, in terms of flexibility, that needs to be acquired from a supplier (a purchasing function). Similar results are observed for the various non-stationary demand patterns.

\subsubsection{Advance Information}

To study the nature of advance information that a buyer provides a supplier using commitments, we first observed the nature of commitments made in period $t$ for $T-1 .^{8}$ Using the OLFC-RHF heuristic, we noticed that commitments decrease over time; that is, commitments for more "immediate" periods are usually larger than those for periods farther into the future. For the static problem, Eqs. (15) and (16) suggest this behavior. Similar behavior then should be expected of the OLFC-RHF heuristic because it solves the static problem in a rolling horizon manner. Furthermore, when operating in a rolling horizon manner, the commitments for more "immediate" periods increase with flexibility. Thus we argue that commitments $Q_{t, t+i}$ are not very reliable pieces of information for a supplier. On the other hand, the buyer is obligated to purchase the minimum number of units given by $\left(1-\underline{\alpha}_{t, t+i}\right) Q_{t, t+i}$. Because this is a guaranteed minimum purchase based on the commitments (and hence the buyer has a financial liability), we claim it is a more reliable indicator of the actual purchase quantity for period $t+i$. We thus modify the measure in (6) to,

$$
\operatorname{MAD}_{t, t+i}=\mathbf{E}\left|\left(1-\underline{\alpha}_{t, t+i}\right) Q_{t, t+i}-Q_{t+i, t+i}\right| .
$$

From a supplier's viewpoint, since the commitments provide advance information regarding actual orders, it is useful to compare $\mathrm{MAD}_{t, T-1}$ with the MAD for the demand and order processes in period $T-1$. For brevity we report here results on $\mathrm{MAD}_{t, T-1}$ only for STATIONARY demand and one value of CV. Similar results were observed for all other cases. Table 4 lists $\mathrm{MAD}_{t, T-1}$ for $t=1, \ldots, T-2$ for $\mathrm{CV}$ of demand equal to 0.25 .

Consider a scenario in which the demand distribution faced by a buyer is unknown to the supplier. In such situations she gets information regarding demands only through the commitments that the buyer gives. It is useful then to compare $\mathrm{MAD}_{t, T-1}$ (shown in Table 4 for $t=1, \ldots, T-2$ ) with the mean absolute deviation of demand (denoted by $\mathrm{MAD}_{d}$ ) for period $T-1$. Recall that we assume demand to be normally distributed. This implies that the standard deviation of demand, $\sigma=1.25 \times \mathrm{MAD}_{d}$. Then for demand CV equal

\footnotetext{
${ }^{8}$ We consider the penultimate period and not the last period to account for any end of horizon effects.
} 
Table 3. Value of an additional 5\% flexibility in the OLFC-RHF contract for STATIONARY demand.

\begin{tabular}{lccc}
\hline & \multicolumn{3}{c}{ Decrease in costs \% } \\
\cline { 2 - 4 } Flexibility \% & $\mathrm{CV}=0.25$ & $\mathrm{CV}=0.33$ & $\mathrm{CV}=0.5$ \\
\hline 5 & 0.83 & 1.07 & 1.44 \\
10 & 0.31 & 0.43 & 0.66 \\
15 & 0.16 & 0.24 & 0.38 \\
20 & 0.10 & 0.15 & 0.25 \\
25 & 0.06 & 0.10 & 0.17 \\
30 & 0.04 & 0.08 & 0.14 \\
35 & 0.03 & 0.05 & 0.11 \\
40 & 0.02 & 0.04 & 0.09 \\
45 & 0.01 & 0.03 & 0.07 \\
50 & 0.01 & 0.02 & 0.05 \\
\hline
\end{tabular}

to $0.25, \mathrm{MAD}_{d}=20$. We observe that when flexibilities are small, $\mathrm{MAD}_{t, T-1}<\mathrm{MAD}_{d}$ suggesting that advance information itself has reduced variability as compared to the demand (we have already seen earlier that the order variability is lower). However, when flexibilities are large, advance information passed on to the supplier through commitments has a variability higher than the variability in original demand (the boldface numbers in Table 4 indicate the boundary for flexibility above which this happens). This suggests that "order processing" (the process that generates commitments/orders) itself creates variability in the information passed on to the supplier, even though the actual orders have variability lower than that of the demand. ${ }^{9}$

Finally, recall the order process variability discussed earlier and shown in Table 1. Assuming the orders are normally distributed, we can compute the MAD of the orders and compare it with the $\mathrm{MAD}_{t, T-1}$ displayed in Table 4. For each level

\footnotetext{
${ }^{9}$ We also measured MAD for upward or downward biases. An upward (downward) bias will be when the commitments are larger (smaller) than actual orders. From our numerical studies, we observe that under the original definition of MAD in Section 3.1 there is an upward bias for every level of flexibility. This can be explained by the relatively high shortage cost that encourages the buyer to commit high to avoid shortages even at the expense of excess inventory. Interestingly the ratio of upward to downward bias is decreasing with flexibility. As flexibility increases the risk of shortages become small but the risk of excess inventory might be high due to the minimum quantity that must be purchased. We observe a similar phenomenon when using the updated definition of MAD. While the trend remains same with increase in flexibility, we observe an upward bias for low flexibility (though at lower levels than with the original MAD definition) which disappears when the flexibility is high. Recall that using the updated definition we compare the actual orders to the lower bound of feasible order quantities and not to the actual commitments. Clearly, because we compare the actual orders to the lower bound, upward bias is less likely as demonstrated by the data. We tested our hypothesis by reducing the shortage cost and indeed the magnitude of the upward bias decreases. Due to space limitations we are unable to provide a complete numerical study of this phenomenon.
}

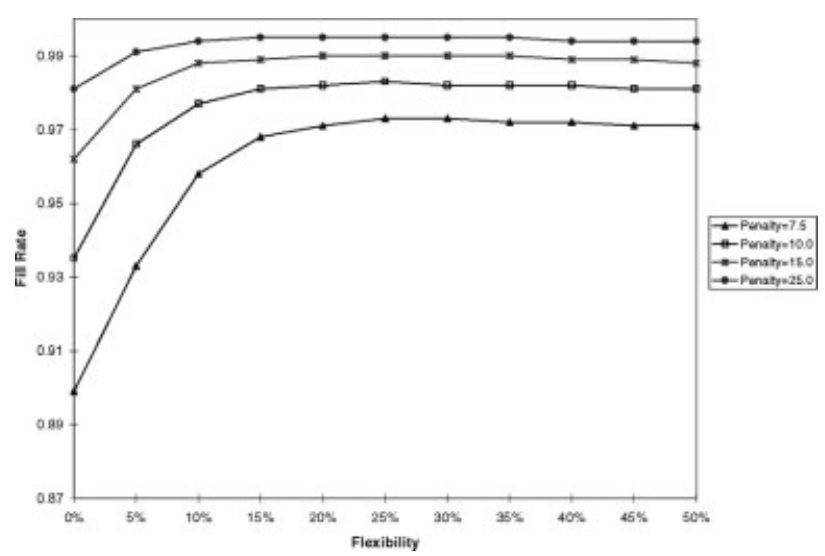

Figure 3. Average fill rate versus flexibility for STATIONARY demand with $\mathrm{CV}=0.50$.

of flexibility we identify the first period in which $\mathrm{MAD}_{t, T-1}$ drops below the MAD of orders for period $T-1$; this is shown in italicized numbers in Table 4. For example, consider the case when demand $\mathrm{CV}=0.25$ and flexibility is $5 \%$. Then MAD of order process is computed to be $8.31 .{ }^{10}$ From Table 4 we observe that the period with $\mathrm{MAD}_{t, T-1}$ closest to 8.31 is period 8 and $\mathrm{MAD}_{t, T-1} \leq 8.31$ for $t>8$. This again suggests that due to order processing, the information passed to the supplier in early periods has larger variability than the order itself. Once we are within 1-3 periods of the time of actual order, the MAD in the information reduces below the MAD for the orders. Recall that for low levels of flexibility, advance information has less variability than the demand. To understand the logic for comparing the MAD of the order process with the CV of demand, first consider the base case of no RHF contract between the buyer and the supplier. We thus have the classical newsboy model in which it is well known that the order process tracks the demand process. So from the supplier's perspective, in absence of any contract, order process variability tracks demand variability. By signing an RHF-type contract, the supplier only hopes to reduce the order process variability further. Hence if the MAD of order process deviates much more than the inherent variability in the demand process, that information is of little value to the supplier.

The above discussion suggests that information within three periods of the actual order is quite informative. Using this insight, we measured $\mathrm{MAD}_{t, t+i}$ for all $t=1, \ldots, T-1$ and $i=1,2$, and 3 for the various demand patterns. As an illustration, results for the triangular demand pattern with a $\mathrm{CV}=0.33$ is shown in Fig. 4 . We make the following observations. The MAD tracks the pattern of

\footnotetext{
${ }^{10}$ This is derived by dividing the standard deviation of the order process (not reported here) by 1.25. The standard deviation of the order process cannot be directly inferred from Table 1 because the average order size was not equal to the mean demand for the finite horizon problems considered here.
} 
Table 4. Mean absolute deviation (MAD) of commitments for STATIONARY demand with $\mathrm{cv}=0.25$.

\begin{tabular}{|c|c|c|c|c|c|c|c|c|c|c|}
\hline \multirow[b]{2}{*}{ Flexibility $\%$} & \multicolumn{9}{|c|}{ Period $t$} & \multirow[b]{2}{*}{10} \\
\hline & 1 & 2 & 3 & 4 & 5 & 6 & 7 & 8 & 9 & \\
\hline 5 & 10.52 & 10.76 & 10.81 & 11.31 & 11.19 & 10.97 & 10.35 & $\underline{8.58}$ & 6.04 & 1.40 \\
\hline 10 & 11.69 & 11.77 & 11.78 & 12.02 & 12.03 & 12.22 & 12.49 & $\overline{11.90}$ & 9.89 & 2.95 \\
\hline 15 & 14.34 & 14.13 & 14.13 & 13.78 & 13.78 & 13.67 & 13.50 & 13.37 & 12.15 & 5.35 \\
\hline 20 & 17.52 & 17.13 & 17.13 & 16.40 & 16.40 & 16.05 & 15.44 & $\overline{15.17}$ & 14.01 & 8.18 \\
\hline 25 & 21.08 & 20.57 & 20.57 & 19.58 & 19.58 & 19.11 & 18.20 & 17.78 & $\overline{16.22}$ & 11.39 \\
\hline 30 & 25.24 & 24.67 & 24.67 & 23.54 & 23.54 & 22.99 & 21.91 & 21.39 & $\overline{19.42}$ & 15.82 \\
\hline 35 & 29.99 & 29.40 & 29.40 & 28.24 & 28.24 & 27.67 & 26.54 & 25.97 & 23.81 & $\overline{20.42}$ \\
\hline 40 & 35.09 & 34.52 & 34.52 & 33.37 & 33.37 & 32.81 & 31.68 & 31.12 & 28.91 & 25.79 \\
\hline 45 & 40.43 & 39.89 & 39.89 & 38.82 & 38.82 & 38.28 & 37.21 & 36.68 & 34.56 & 31.38 \\
\hline 50 & 45.88 & 45.38 & 45.38 & 44.39 & 44.39 & 43.89 & 42.90 & 42.41 & 40.44 & 37.26 \\
\hline
\end{tabular}

nonstationarity. This is because while the mean demand follows a TRIANGULAR pattern, the $\mathrm{CV}$ of demand is fixed and hence the standard deviation of demand also follows a TRIANGULAR pattern. Because MAD is closely related to the standard deviation of demand, it too follows a triangular pattern. Furthermore, the pattern is dampened as (i) we get closer to the period of the order for a given flexibility (MAD with 1-period window is flatter than with a 3-period window), and (ii) flexibility reduces for a given window (MAD for $10 \%$ flexibility is more flat as compared to the MAD for $50 \%$ flexibility for a 1 or 3-period window).

\subsection{RHF versus ZLF: A Buyer-Supplier Perspective}

Recall that the two primary motivations for a supplier to offer an RHF contract are reduction in order process variability and the availability of advance information regarding orders. Although a buyer could use any of the heuristics presented to evaluate an RHF contract, based on our computational studies it is likely that when the salvage value is low the buyer will use the ZLF-UB heuristic because it results in lower expected costs. In addition, this heuristic results in a lower variability of the order process; thus a supplier would also prefer the order process of ZLF-UB to that of the OLFC-RHF heuristic. Therefore, for low salvage values the

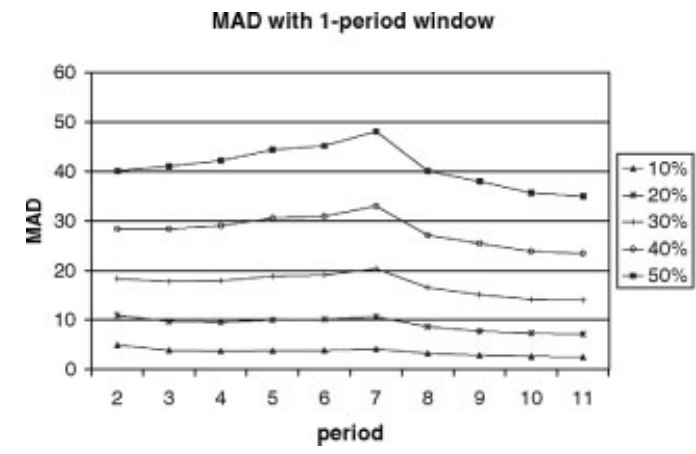

ZLF-UB heuristic dominates the OLFC-RHF heuristic for both players.

On the other hand, when salvage values are high a buyer prefers the OLFC-RHF heuristic over the ZLF-UB. We have already seen the effect of the OLFC-RHF heuristic on the order process variability and advance information. We also studied the effect of the ZLF-UB heuristic on the order process variability. From Table 1 we see that the ZLF-UB heuristic often results in a smaller ORCV for any level of flexibility. This is expected since for the OLFC-RHF heuristic with, say, $5 \%$ flexibility, the effective flexibility in the later periods is much larger. Thus the supplier prefers the order process of a ZLF-UB heuristic over that of the OLFC-RHF heuristic. Therefore for high salvage values neither heuristic dominates.

However, assuming that the original flexibility is $\alpha$, we find that in many cases there is a flexibility $\widetilde{\alpha}(\widetilde{\alpha}>\alpha)$ such that the expected cost of using OLFC-RHF with $\alpha$ is equal to the expected cost of using ZLF-UB with $\widetilde{\alpha}$. Furthermore, the variability of the order process using ZLF-UB (with $\widetilde{\alpha}$ ) is significantly smaller than that obtained by using OLFCRHF (with $\alpha$ ). For example, with stationary demand at CV = 0.5 and salvage value equals purchase cost, we observe that the expected cost to a buyer using the OLFC-RHF heuristic to solve an RHF contract with $10 \%$ flexibility is equal to the expected cost of a ZLF contract with $17 \%$ flexibility.

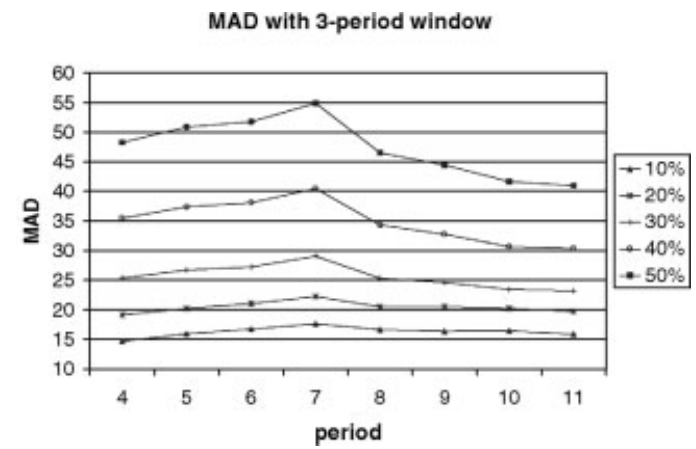

Figure 4. MAD of order with a one and three period before the order for TRIANGULAR demand with CV $=0.33$. 
So the buyer should be indifferent between an RHF contract with $10 \%$ flexibility and a ZLF contract with $17 \%$ flexibility. However, the order process CV for a $10 \%$ flexibility RHF contract is 0.20 (using an OLFC-RHF heuristic) while that for a $17 \%$ flexibility ZLF contract is 0.12 . So the supplier gains with a ZLF contract in this situation. Thus a ZLF contract with $17 \%$ flexibility weakly dominates an RHF contract with $10 \%$ flexibility. This leads us to believe that both the buyer and the supplier are better off signing a ZLF contract with the appropriate flexibility even when salvage values are high.

For nonstationary demands, the nonstationarity of the ORCV itself makes the comparison between OLFC-RHF and ZLF-UB difficult. We do observe that for low-medium flexibility, the ORCV pattern for ZLF-UB dominates that under OLFC-RHF. In general, however, for such situations comparisons will critically depend on the exact cost structure of the supplier, which is beyond the scope of the present work.

To summarize, the order process under a ZLF-UB heuristic is less variable than the order process under a OLFC-RHF heuristic. On the other hand, for high salvage values the OLFC-RHF heuristic results in lower costs for the buyer. Other heuristics that balance the advantages of the two presented here is potentially an area of further research. ${ }^{11}$

\section{SUMMARY AND CONCLUSION}

In this article, we study a class of contracts called the RHF contract used as an instrument to share forecast information and risk between a buyer and a supplier. Under such contracts, at the beginning of the horizon a buyer makes commitments for components for each period. The supplier in turn provides limited flexibility to adjust the current order and update the future commitments in a rolling horizon manner. We present a general model for an RHF contract and propose two measurements for the order process that capture the variability in the order process and advance information shared between the supplier and buyer through the commitments. The optimal policy for the general model is unknown. We present

\footnotetext{
${ }^{11}$ It should be rather straighforward too see that a combination of the the rolling horizon OLFC-RHF and ZLF-UB heuristics would give superior results. In Appendix D we present such a heuristic called the OLFC-ZLF heuristic. The new heuristic is essentially a rolling horizon implementation of ZLF-UB heuristic. Computing OLFC-ZLF heuristic solution for a demand scenario (the demand realizations in each period as we move forward in a horizon) is not time consuming; it is approximately the horizon length times the time to compute the optimal ZLF - see discussion in Appendix C. Therefore, it is amenable to implementation. To test its efficacy, however, requires computation of expected costs of the OLFC-ZLF heuristic (important for comparing the performance of these heuristics and for sensitivity analyses), a time consuming task since we need to average over numerous demand scenarios.
}

two heuristics and a lower bound. Using extensive computational studies, we then demonstrate the effectiveness of the heuristics and provide several managerial insights into the properties of RHF contracts. For example, we show that (a) often "unlimited" flexibility offered by a newsvendor model is unnecessary, (b) low levels of flexibility is sufficient to match the performance of a newsvendor model, (c) there are decreasing returns to flexibility, (d) larger flexibilities allow a buyer to offer higher service levels (measured by fill-rate), (e) variability in the order process is lower than the variability in the demand process, (f) mean absolute deviation of the commitment from actual order decreases as we get closer to the period in which orders are placed, and (g) "order processing" creates variability in the order process that is sometimes larger than the variability in the original demand. Finally, we illustrate that for several cases a ZLF contract (which is a subclass of RHF contract) is efficient in that it provides a buyer with the same expected cost as in a more general RHF contract (under the OLFC-RHF heuristic) and simultaneously provides a supplier with lower variability in the order process.

Our computational studies consider stationary and nonstationary demands without any forecasting of market demand. We believe that from an analytical perspective, the heuristics and the lower bound will still be correct with a slight modification. The ZLF-UB heuristic and the lower bounds that follows a modified base stock policy will now have to incorporate the most recent demand realization in its state vector. We also believe that all the key insights generated will hold for the case with demand forecasting.

The analysis presented here is a first step in understanding contracts that require commitments with limited flexibility. We have analysed a contract for a single product in a single-stage setting. Extensions of similar analyses to multiple products with/without component commonality are also areas of further research.

Finally, the focus of our paper has solely been on the analysis of a contract presented to the buyer. We have demonstrated its potential benefits to the supplier. Will such contracts arise in a competitive setting that also includes the supplier as a decision maker? From a supplier's perspective, minimum orders guarantee business continuity; offering flexibility allows it to exploit any upside potential that may arise. Of course, the flexibility offered needs to be balanced with the resulting impact (through order variability) on its cost structure. Some papers in the literature have addressed the contracting problem leading to contracts of the nature described here, albeit in a single period (multi-stage) situation; see for example, Tsay [20] and Barnes-Schuster et al. [5] for justification on the basis of channel coordination. These papers, however, do not have a supplier cost model that is impacted by order process variability; we conjecture that in a model that has a supplier with such costs, one would see an RHF type contract arising in equilibrium. 


\section{APPENDIX A: ALGORITHMS}

\section{OLFC-RHF Heuristic}

Algorithm 1:

1. Set $j=1$.

2. Find $x_{j}$ such that $\left.\sum_{i=j}^{T} \frac{\partial J}{\partial Q_{1}(i)}\right|_{x_{j}}=0$.

3. Find $S_{j}$. (This requires solving a single period "newsvendor" problem)

4. If $S_{j} \leq x_{j}$ then

$$
\begin{aligned}
& \text { set } S_{j}^{*}=S_{j} . \\
& \text { If } j=T \text { then STOP. } \\
& \text { else } j=j+1 \text {; GOTO } 2 .
\end{aligned}
$$

else

set $S_{i}^{*}=x_{j}, i=j, j+1, \ldots, T$

$k^{*}=j$. STOP

Algorithm 2:

1. Set $i=0$ and $\triangle_{i}=0$, for $i=0, \ldots, T-t$. The OLFC optimal solution for periods $t-1, \ldots, T$ obtained in period $t-1$ is denoted by $Q_{t-1, t-1}^{*}, \ldots, Q_{t-1, T}^{*}$.

2. Solve a relaxed version of the problem in (9)-(11) (ignoring constraints (11)). The relaxed problem can be solved by Algorithm 1. Let the solution of the relaxed problem (for period t) be $\tilde{Q}_{t, t}, \ldots, \tilde{Q}_{t, T}$.

3. If $\left(1-\alpha_{t, t+i}\right) Q_{t-1, t+i}^{*} \leq \tilde{Q}_{t, t+i} \leq\left(1+\alpha_{t, t+i}\right) Q_{t-1, t+i}^{*}$ then set $Q_{t, t+i}^{*}=\tilde{Q}_{t, t+i} ; i=i+1$; Go то 3 .

else If $\left(1-\alpha_{t, t+i}\right) Q_{t-1, t+i}^{*} \geq \tilde{Q}_{t, t+i}$ then

set $\triangle_{i}=\tilde{Q}_{t, t+i}-\left(1-\alpha_{t, t+i}\right) Q_{t-1, t+i}^{*}$;

$Q_{t, t+i}^{*}=\left(1-\alpha_{t, t+i}\right) Q_{t-1, t+i}^{*}$;

$\tilde{Q}_{t, t+i+1}=\triangle_{i}+\tilde{Q}_{t, t+i+1}$

If $i=T$ then STOP;

else $i=i+1$; Go To 3 ;

else set $\triangle_{i}=\tilde{Q}_{t, t+i}-\left(1+\alpha_{t, t+i}\right) Q_{t-1, t+i}^{*}$;

$Q_{\tilde{Q}, t+i}^{*}=\left(1+\alpha_{t, t+i}\right) Q_{t-1, t+i}^{*}$

$\tilde{Q}_{t, t+i+1}=\triangle_{i}+\tilde{Q}_{t, t+i+1}$

If $i=T$ then STOP;

else $i=i+1$; Go To 3 ;

\section{Algorithm 3:}

1. Solve the static problem using Algorithm 1. Let the solution of this problem be $Q_{1,1}^{0}, \ldots, Q_{1, T}^{0}$.

2. Set $j=1$.

3. Solve problem (P2) to get $Z_{1}^{j}, \ldots, Z_{T}^{j}$ given $Q_{1,1}^{j-1}, \ldots, Q_{1, T}^{j-1}$. This can be done by a standard backward DP.

4. Solve problem (P3) to get $Q_{1,1}^{j}, \ldots, Q_{1, T}^{j}$.

5. If "termination condition is satisfied" then STOP; otherwise $j=$ $j+1$; GOTO 3 .

\section{APPENDIX B: PROOFS}

PROOF OF LEMMA 4.1: We first write (8) as:

$$
\begin{aligned}
& \min _{Q_{1}(1), \ldots, Q_{1}(T)} J\left(Q_{1}(1), \ldots, Q_{1}(T)\right) \\
& \text { s.t. } Q_{1}(1) \geq 0 \\
& Q_{1}(i+1) \geq Q_{1}(i) \quad \forall i=1, \ldots, T-1
\end{aligned}
$$

The Lagrangian relaxation of (B1) can be written as:

$\mathcal{L}\left(Q_{1}(1), \ldots, Q_{1}(T)\right)=J\left(Q_{1}(T), \ldots, Q_{1}(T)\right)-\sum_{i=0}^{T-1} \lambda_{i}\left(Q_{1}(i+1)-Q_{1}(i)\right)$,

where $\lambda_{i}$ is the Lagrange multiplier associated with the constraint $Q_{1}(i+$ 1) $\geq Q_{1}(i), i=1, \ldots, T-1$ and $\lambda_{0}=0$. The partial derivatives of the Lagrangian function are:

$$
\frac{\partial \mathcal{L}}{\partial Q_{1}(i)}=(h+p) F_{1, i}\left(Q_{1}(i)\right)-p+\lambda_{i}-\lambda_{i-1} \quad \text { for } i=1, \ldots, T-1
$$

and

$$
\frac{\partial \mathcal{L}}{\partial Q_{1}(T)}=(h-s+p) F_{1, T}\left(Q_{1}(T)\right)-p+\lambda_{T-1}
$$

To solve the problem we must find $Q_{1}(1), \ldots, Q_{1}(T)$ and $\lambda_{1}, \ldots, \lambda_{T}$ such that:

1. The $T$ partial derivatives of the Lagrangian function, given by (B2)-(B3) are equal to zero.

2. $Q_{1}(i+1) \geq Q_{1}(i)$ for $i=0, \ldots, T-1$.

3. $\lambda_{i}\left(Q_{1}(i+1)-Q_{1}(i)\right)=0$ for $i=0, \ldots, T-1$.

Part 1: Since $S_{1} \leq S_{2} \leq \cdots \leq S_{T-1} \leq S_{T}$ the constraints in (8) are all satisfied. We set the multipliers, $\lambda_{i}$ to zero and $Q_{1}(i)=S_{i}$. Substituting into (B2) and (B3) we see that the Kuhn-Tucker (K-T) conditions for optimality are satisfied.

Part 2: Suppose $S_{T}<S_{T-1}$. We will show that the optimal solution has the property that there exists a $k^{*}<T$ such that for every period $j>k^{*}$ the periodical commitment quantity is equal to zero. Algorithm 1 (see Appendix A) determines the optimal value of $k^{*}$. We make the following two observations:

1. The static problem given by (8) is convex and thus all the partial derivatives are monotone non-decreasing.

2. Let $S_{i}^{*}, i=1, \ldots, T$ be the optimal solution of the static problem. Then, by summing the derivatives in (B2) and (B3) we have $\left.\sum_{i=1}^{T} \frac{\partial J}{\partial Q_{1}(i)}\right|_{S_{i}^{*}}=0$.

To prove part 2 of the lemma, we first prove the existence of $k^{*}<T$ such that $S_{j}>x_{j}$ for $j \geq k^{*}$. By contradiction, suppose such a $k^{*}$ does not exist. Then we get $S_{j} \leq x_{j}$ for $j=1, \ldots, T-1$. From the definition of $x_{T-1}$ (see step 2 of Algorithm 1) we get

$$
\left.\frac{\partial J}{\partial Q_{1}(T)}\right|_{x_{T-1}}+\left.\frac{\partial J}{\partial Q_{1}(T-1)}\right|_{x_{T-1}}=0 .
$$

Observe that $x_{T-1} \geq S_{T-1}>S_{T}$; the first inequality is due to the contradiction assumption and the second inequality is due to the condition assumed in the Lemma. Observe that $S_{T}$ and $S_{T-1}$ in (12) and (13) solve $\left.\frac{\partial J}{\partial Q_{1}(T)}\right|_{S_{T}}=0$ and $\left.\frac{\partial J}{\partial Q_{1}(T-1)}\right|_{S_{T-1}}=0$ respectively. But since $x_{T-1} \geq S_{T-1}>S_{T}$, we get $\left.\frac{\partial J}{\partial Q_{1}(T)}\right|_{x_{T-1}} \geq 0$ and $\left.\frac{\partial J}{\partial Q_{1}(T-1)}\right|_{x_{T-1}}>0$ which contradicts (B4). Thus the existence of $k^{*}<T$ such that $S_{j}>x_{j}$ for $j \geq k^{*}$ is guaranteed. To prove the optimality of the periodical commitments in (14) we show that the commitments $Q_{1, i}$ are feasible and satisfy the K-T conditions (B2) and (B3).

To ensure feasibility we need to show that $Q_{1}(i) \geq Q_{1}(i-1)$ for all $i=$ $1, \ldots, T$. Observe that $S_{1} \leq, \ldots, \leq S_{k^{*}-1}$, and thus $Q_{1}(i)-Q_{1}(i-1) \geq 0$

Naval Research Logistics DOI 10.1002/nav 
for $i=1, \ldots, k^{*}-1$. Since $S_{k^{*}-1} \leq x_{k^{*}-1}$ (from the definition of $k^{*}$ ) we have

$$
\left.\frac{\partial J}{\partial Q_{1}\left(k^{*}-1\right)}\right|_{x_{k^{*}-1}} \geq\left.\frac{\partial J}{\partial Q_{1}\left(k^{*}-1\right)}\right|_{S_{k^{*}-1}}=0 .
$$

But $x_{k^{*}-1}$ solves

$$
\left.\sum_{i=k^{*}-1}^{T} \frac{\partial J}{\partial Q_{1}(i)}\right|_{x_{k^{*}-1}}=0
$$

This implies that

$$
\left.\sum_{i=k^{*}}^{T} \frac{\partial J}{\partial Q_{1}(i)}\right|_{x_{k^{*}-1}}<0
$$

But

$$
\left.\sum_{i=k^{*}}^{T} \frac{\partial J}{\partial Q_{1}(i)}\right|_{x_{k^{*}}}=0
$$

Convexity of $J(\cdot)$ then implies that $x_{k^{*}} \geq x_{k^{*}-1} \geq S_{k^{*}-1}$. This completes the feasibility proof. We now prove parts 2 and 3 of the Lemma to show that Algorithm 1 results in the optimal solution.

Case $1\left(j<k^{*}\right)$ : The partial derivatives of the Lagrangian

$$
\left.\frac{\partial \mathcal{L}}{\partial Q_{1}(j)}\right|_{S_{j}}=(h+p) F_{1, j}\left(S_{j}\right)-p=0
$$

with $\lambda_{j}=0$. The multipliers are non-negative and $\lambda_{j}\left(Q_{1}(j+1)-Q_{1}(j)\right)=$ 0 for $j=0, \ldots, k^{*}-1$.

Case $2\left(j=k^{*}\right)$ : For $j=k^{*}, \lambda_{j}=-\left.\frac{\partial J}{\partial Q_{1}(j)}\right|_{x_{j}}$. Since $\left.\frac{\partial J}{\partial Q_{1}(j)}\right|_{S_{j}}=0$, $x_{j}<S_{j}$ and $J(\cdot)$ is convex, it is clear that $\lambda_{j} \geq 0$. Thus all K-T conditions are satisfied.

Case $3\left(k^{*}<j \leq T\right)$ : For $j=k^{*}+1, \ldots, T-1$ we set (refer to (B2))

$$
\lambda_{j}=-\left.\frac{\partial J}{\partial Q_{1}(j)}\right|_{x_{j}}+\lambda_{j-1} .
$$

Now, $\left.\frac{\partial J}{\partial Q_{1}(j)}\right|_{x_{j}} \leq 0$ implies that $\lambda_{j} \geq 0$. From (12) and (13) we have that

$$
\lambda_{T-1}=-\left.\frac{\partial J}{\partial Q_{1}(T)}\right|_{x_{j}} \geq 0 .
$$

Again, all K-T conditions are satisfied and we have the optimal solution to the static problem as described.

\section{PROOF OF PROPOSITION 4.2:}

Consider the restrictions on the order quantity $Q_{t, t}$ in period $t$ under the RHF contract,

$$
\left(1-\underline{\alpha}_{t-1, t}\right) Q_{t-1, t} \leq Q_{t, t} \leq\left(1+\bar{\alpha}_{t-1, t}\right) Q_{t-1, t} .
$$

Now the restrictions on the commitment for period $t$ in period $t-1, Q_{t-1, t}$ were

$$
\left(1-\underline{\alpha}_{t-2, t}\right) Q_{t-2, t} \leq Q_{t-1, t} \leq\left(1+\bar{\alpha}_{t-2, t}\right) Q_{t-2, t} .
$$

Substituting (B6) into (B5), we write,

$$
\left(1-\underline{\alpha}_{t-2, t}\right)\left(1-\underline{\alpha}_{t-1, t}\right) Q_{t-2, t} \leq Q_{t, t} \leq\left(1+\bar{\alpha}_{t-2, t}\right)\left(1+\bar{\alpha}_{t-1, t}\right) Q_{t-2, t} .
$$

Observe that (B7) gives higher level of flexibility for $Q_{t, t}$ than given by (B5). Substituting bounds for $Q_{t-2, t}, \ldots, Q_{1, t}$ into (B7) iteratively, we write,

$$
\prod_{k=1}^{t}\left(1-\underline{\alpha}_{k, t}\right) Q_{1, t} \leq Q_{t, t} \leq \prod_{k=1}^{t}\left(1+\bar{\alpha}_{k, t}\right) Q_{1, t}
$$

which is equivalent to $\left(1-\underline{\gamma}_{t, t}\right) Q_{1, t} \leq Q_{t, t} \leq\left(1+\bar{\gamma}_{t, t}\right) Q_{1, t}$. Since (B8) gives a higher level of flexibility in choosing $Q_{t, t}$ as compared to (B5), the ZLF-LB contract provides a lower bound.

PROOF OF PROPOSITION 5.1: Observe that Problem (P2) as defined by (17)-(18) is similar to the classical capacitated inventory problem. In the capacitated inventory problem the lower bound on the order quantity is always zero and the upper bound is always the capacity; in contrast, for Problem (P2) with non-zero commitments, the actual order quantity in a period will have a finite non-negative lower and upper bounds as described by (18). Federgruen and Zipkin [13] proved the optimality of a modified base stock policy for the capacitated inventory problem. The proof follows along similar lines.

PROOF OF LEMMA 5.2: The constraints (18) can be written as $A X=$ $b$ where

$$
A=\left[\begin{array}{cccc}
1 & 0 & 1 & 0 \\
1 & 0 & 0 & -1
\end{array}\right]
$$

the vector $X=\left[Q_{i, i}, Q_{i, i}, u_{i}, l_{i}\right]$ with $u_{i}, l_{i} \geq 0$ as the slack and surplus variables and the vector $b=\left[\left(1+\bar{\alpha}_{i, i}\right) Q_{1, i},\left(1-\underline{\alpha}_{i, i}\right) Q_{1, i}\right]$. Using the fact that (P2) is convex and applying Theorem 5.7 of Rockafellar [17] we get convexity of $A C_{1}^{*}\left(x_{1}, Q_{1,1}, \ldots, Q_{1, T}\right)$ in $\left(x_{1}, Q_{1,1}, \ldots, Q_{1, T}\right)$.

PROOF OF PROPOSITION 5.2: Observe that

$$
B C_{1}^{j}\left(x_{1}, Q_{1,1}^{j-1}, \ldots, Q_{1, T}^{j-1} \mid Z_{1}^{j}, \ldots, Z_{T}^{j}\right)=A C_{1}^{* j}\left(Q_{1,1}^{j-1}, \ldots, Q_{1, T}^{j-1}\right) .
$$

After solving (P3) at iteration $j$, we get

$$
B C_{1}^{* j}\left(x_{1}, Q_{1,1}^{j}, \ldots, Q_{1, T}^{j} \mid Z_{1}^{j}, \ldots, Z_{T}^{j}\right) \leq A C_{1}^{* j}\left(x_{1}, Q_{1,1}^{j-1}, \ldots, Q_{1, T}^{j-1}\right) .
$$

Subsequently, solving problem (P2) given $\left(Q_{1,1}^{j}, \ldots, Q_{1, T}^{j}\right)$ we get

$$
A C_{1}^{* j+1}\left(x_{1}, Q_{1,1}^{j}, \ldots, Q_{1, T}^{j}\right) \leq B C_{1}^{* j}\left(x_{1}, Q_{1,1}^{j}, \ldots, Q_{1, T}^{j} \mid Z_{1}^{j}, \ldots, Z_{T}^{j}\right)
$$

Thus we get that $B C_{1}^{* j+1}(\cdot \mid \cdot) \leq A C_{1}^{* j+1}(\cdot) \leq B C_{1}^{* j}(\cdot \mid \cdot)$ for every $j$. Since the cost functions are bounded from below by zero it is clear that the algorithm converges. We now prove, by contradiction, that the algorithm converges to the optimal solution of problem (ZLF). Suppose that the algorithm converges to $\tilde{Q}_{1,1}, \ldots, \tilde{Q}_{1, T}, \tilde{Z}_{1}, \ldots, \tilde{Z}_{T}$ whereas the optimal solution to the problem (ZLF) is $Q_{1,1}^{*}, \ldots, Q_{1, T}^{*}, Z_{1}^{*}, \ldots, Z_{T}^{*} \neq$ $\tilde{Q}_{1,1}, \ldots, \tilde{Q}_{1, T}, \tilde{Z}_{1}, \ldots, \tilde{Z}_{T}$. Before, we show the last step, we need the following. To show the dependence of $A C_{1}^{*}(\cdot)$ on the commit vector and the 
optimal modified base stock solution (the vector of $Z$ ) explicitly, we write $A C_{1}^{* j}\left(x_{1}, Z_{1}, \ldots, Z_{T} \mid Q_{1,1}, \ldots, Q_{1, T}\right) \equiv A C_{1}^{* j}\left(x_{1}, Q_{1,1} \ldots, Q_{1, T}\right)$. Then,

$$
\begin{aligned}
& A C_{1}^{*}\left(x_{1}, Z_{1}^{*}, \ldots, Z_{T}^{*} \mid Q_{1,1}^{*}, \ldots, Q_{1, T}^{*}\right) \\
& <A C_{1}^{*}\left(x_{1}, \tilde{Z}_{1}, \ldots, \tilde{Z}_{T} \mid \tilde{Q}_{1,1}, \ldots, \tilde{Q}_{1, T}\right) \\
& \quad=B C_{1}^{j}\left(x_{1}, \tilde{Q}_{1,1}, \ldots, \tilde{Q}_{1, T} \mid \tilde{Z}_{1}, \ldots, \tilde{Z}_{T}\right)
\end{aligned}
$$

But inequality (B12) is a contradiction to the convexity of problems ZLF, P2, and P3. Thus (B12) does not hold which proves that the algorithm converges to the optimal solution.

\section{APPENDIX C: IMPLEMENTATION OF THE HEURISTICS AND LOWER BOUND}

\section{ZLF Contract}

Algorithm 2: Observe that problem (P2) can be easily solved by using standard Dynammic Programming (DP) techniques. The only difference between the standard newsvendor problem and (P2) is that in problem (P2) the order quantities, $Q_{i, i}$ are constrained by the periodical commitments $Q_{1,1}, \ldots, Q_{1, T}$ given by (18). In solving problem (P2) we proceed exactly in the same way as if we solve the standard newsvendor problem using backward DP. In the period $i$, to solve for $Z_{i}$ we assume knowledge of the $T-i$ modified base-stock levels for periods $i+1, \ldots, T$ and $A C_{i+1}^{*}\left(x_{i+1}, Q_{1, i+1}, \ldots, Q_{1, T}\right)$, the optimal expected costs from period $i+1$ through $T$. The modified base-stock level for period $i, Z_{i}$ is then calculated assuming that period $i$ is unconstrained. Subsequently, the new cost-to-go function $A C_{i}^{*}\left(x_{i}, Q_{1, i}, \ldots, Q_{1, T}\right)$ is constructed. Observe that the cost-togo function, $A C_{i}^{*}\left(x_{i}, Q_{1, i}, \ldots, Q_{1, T}\right)$, will be affected by the constraints in period $i$. Solving for the modified base stock level $Z_{i}$ for period $i$ assuming that the order quantity in period $i$ is not constrained is justified by the fact that the cost function $A C_{i}(\cdot)$ is convex (see Lemma 5.2) and the constraints are linear. Thus it is always optimal to order either up to the unconstrained base stock level or quantities specified by the bounds.

Algorithm 3: For the ZLF contract, we implement Algorithm 3 to compute the optimal commitment quantities and the corresponding adjustment policy for given parameters. While the implementation of Algorithm 1 and steps 2 and 3 of Algorithm 3 are straightforward, we elaborate on implementation of step 4 (updating the commitment vector). In step 4 of algorithm 2, we solve (P3) to update the commit vector for the adjustment policy computed earlier in step 3. Recall that we update the commit vector $\left(Q_{1,1}, \ldots, Q_{1, T}\right)$ using (21) working backward from period $T$. The only problem in solving (21) is to determine the starting inventory position $x_{i}$ for period $i$. We do this as follows. Recall that we have the various demand points generated for each period's demands (see discussion just before subsection 6.1 on Monte Carlo integration for computing first partials). We use these demand points (for periods 1 to $i-1$ ) to generate sample realizations of starting inventory $x_{i}$ for period $i$. This is easily done since we know the optimal policy structure. For any given value of the minimizer $Q_{i, i}$ in (21), we then compute the expected cost $B C_{i}$ using the sample realizations $x_{i}$ and demand realizations for period $i+1$ to $T$. Finally, searching over $Q_{i, i}$ gives us an update for the commitment in period $i$. Clearly, this step of updating the commit vector $Q$ (step 4 of algorithm 3 ) is the most computationally intensive of all the steps. Our computational studies indicate that the first iteration of Algorithm 3 takes about 1.5 seconds, whereas each subsequent iteration of step 4 and 3 of algorithm 2 take approximately one minute depending upon the number of points used in the Monte Carlo simulation. We observe that the expected costs converge within 4 to 5 iterations of Steps 3-4 of algorithm 3 when we start with the commit vector which solves the static problem. We used 500 demand points for each iteration but the last; in the last period we use 2500 demand points. However, we also observe that the solution obtained using steps 2 and 3 of Algorithm 3 alone is within $4 \%$ of the optimal solution for the ZLF model. The difference is lower for low flexibilities.

\section{APPENDIX D: OLFC-ZLF HEURISTIC}

We now combine the rolling horizon OLFC-RHF and ZLF-UB heuristics to give the OLFC-ZLF heuristic. Observe that using the ZLF-UB heuristic, the buyer gives an initial commitment $Q_{1,1}, \ldots, Q_{1, T}$ in period one and subsequently never updates this initial commitment. Now consider the following rolling horizon implementation of the ZLF-UB heuristic.

1. Compute the initial commitment as in the ZLF-UB heuristic. This gives us $Q_{1,1}, \ldots, Q_{1, T}$.

2. In any period $t$, after observing the initial inventory, recompute the optimal policy for the ZLF-UB contract under constraints $\left(1-\underline{\beta}_{t, i}\right) Q_{t-1, i} \leq Q_{t, i} \leq\left(1+\bar{\beta}_{t, i}\right) Q_{t-1, i}$ for $i=t, \ldots, T$.

The difference between the OLFC-RHF heuristic and this rolling horizon implementation of the ZLF-UB heuristic is that in a ZLF-UB heuristic we compute the optimal commitment (assuming that the buyer will have flexibility to later adjust the actual orders in a future period) whereas in the OLFC-RHF heuristic we ignore this to compute an open loop feedback control solution. We refer to the solution obtained from a rolling horizon implementation of the ZLF-UB contract as the OLFC-ZLF heuristic.

\section{ACKNOWLEDGMENTS}

We wish to thank the associate editor and the two referees for their constructive comments. An earlier version of this manuscript was titled "Analysis of supply Contracts with Forecasts and Flexibility", date March 1995. We wish to thank Sridhar Tayur for his valuable comments on an earlier version of this manuscript.

\section{REFERENCES}

[1] R. Anupindi, Supply management under uncertainty, PhD Thesis, Graduate School of Industrial Administration, Carnegie Mellon University, 1993.

[2] R. Anupindi and R. Akella, An inventory model with commitments, Working paper, J.L. Kellogg Graduate School of Management, Northwestern University, Evanston, IL, October 1993.

[3] R. Anupindi and Y. Bassok, Approximations for multiproduct contracts with stochastic demands and business volume discounts: Single supplier case, IIE Trans 30 (1998), 723-734.

[4] R. Anupindi and Y. Bassok, "Supply contracts with quantity commitments and stochastic demand," Quantitative models for supply chain management, S. Tayur, M. Magazine, and R. Ganeshan (Editors), Kluwer Academic Publishers, Norwell, MA, 1998, pp. 197-232.

[5] D. Barnes-Schuster, Y. Bassok, and R. Anupindi, Coordination and flexibility in supply contracts with options, J Manuf Service Oper Management 4 (2002), 171-207.

[6] Y. Bassok and R. Anupindi, Analysis of supply contracts with total minimum commitment, IIE Trans 29 (1997), 373-381.

[7] Y. Bassok and R. Anupindi, Analysis of supply contracts with commitments and flexibility, University of Michigan, Ann Arbor, MI, 2008

[8] Y. Bassok, R. Srinivasan, A. Bixby, and H. Wiesel, Design of component supply contracts with commitment revision flexibility, IBM J Res Dev 41 (1997), 693-703. 
[9] D.P. Bertsekas, Dynamic programming: Deterministic and stochastic models, Prentice Hall, Englewood Cliffs, NJ, 1987.

[10] F. Chen and D. Krass, Analysis of supply contracts with minimum total commitment and non-stationary demands, Eur J Oper Res 131 (2001), 309-323.

[11] Y. Chen, J.K. Ryan, and D. Simchi-Levi, The impact of exponential smoothing forecasts on the bullwhip effect, Naval Res Logist 47 (2000), 269-286.

[12] D. Farlow, G. Schmidt, and A. Tsay, Supplier management at sun microsystems, 1995.

[13] A. Federgruen and P. Zipkin, An inventory model with limited production capacity and uncertain demands. II. Discounted cost criterion, Math Oper Res 11 (1986), 208-215.

[14] D.E. Knuth, The art of computer programming, Vol. 2: Seminumerical algorithms, 2nd ed., Addison-Wesley, Reading, MA, 1981.

[15] H.L. Lee, V. Padmanabhan, and S. Whang, Information distortion in a supply chain: The bullwhip effect, Management Sci 43 (1997), 546-558.
[16] K. Moinzadeh and S. Nahmias, Adjustment strategies for a fixed delivery contract, Oper Res 48 (2000), 408-423.

[17] R. Rockafellar, Convex analysis, Princeton University Press, Princeton, NJ, 1970.

[18] A. Scheller-Wolf and S. Tayur, Reducing international risk through quantity contracts Working Paper, GSIA, Carnegie Mellon University, Pittsburgh, PA, 1997.

[19] A. Tal, B. Golany, A. Nemirovski, and J. Vial, Supplierretailer flexible commitments contracts: A robust optimization approach, Faculty of Industrial Engineering and Management, Technion, Israel, 2003.

[20] A. Tsay, The quantity flexibility contract and suppliercustomer incentives, Management Sci 45 (1999), 1339-1358.

[21] A. Tsay and W. Lovejoy, Quantity flexibility contracts and supply chain performance, J Manuf Service Oper Management 1 (1999), 89-111.

[22] A. Tsay, S. Nahmias, and N. Agrawal, "Modeling supply chain contracts: A review," Quantitative models for supply chain management, S. Tayur, M. Magazine, and R. Ganeshan (Editors), Kluwer Academic Publishers, Norwell, MA, 1998, pp. 299-336. 